\title{
Condition for low-mass star formation in shock-compressed metal-poor clouds
}

\author{
Daisuke Nakauchi1 ${ }^{1 \star}$, Kazuyuki Omukai ${ }^{1}$, and Raffaella Schneider ${ }^{2,3}$ \\ ${ }^{1}$ Astronomical Institute, Tohoku University, Aoba, Sendai 980-8578, Japan \\ ${ }^{2}$ Dipartimento di Fisica, Universitá di Roma 'La Sapienza', P.le Aldo Moro 2, I-00185 Roma, Italy \\ ${ }^{3}$ INAF/Osservatorio Astronomico di Roma, Via Frascati 33, 00078, Monte Porzio Catone, Roma
}

Accepted XXX. Received YYY; in original form ZZZ

\begin{abstract}
Shocks may have been prevalent in the early Universe, associated with virialization and supernova explosions, etc. Here, we study thermal evolution and fragmentation of shock-compressed clouds, by using a one-zone model with detailed thermal and chemical processes. We explore a large range of initial density $\left(1-10^{5} \mathrm{~cm}^{-3}\right)$, metallicity $\left(0-10^{-2} Z_{\odot}\right)$, UV strength (0-500 times Galactic value), and cosmic microwave background temperature (10 and $30 \mathrm{~K})$. Shock-compressed clouds contract isobarically via atomic and molecular line cooling, until self-gravitating clumps are formed by fragmentation. If the metals are only in the gas-phase, the clump mass is higher than $\sim 3 \mathrm{M}_{\odot}$ in any conditions we studied. Although in some cases with a metallicity higher than $\sim 10^{-3} \mathrm{Z}_{\odot}$, re-fragmentation of a clump is caused by metal-line cooling, this fragment mass is higher than $\sim 30 \mathrm{M}_{\odot}$. On the other hand, if about half the mass of metals is condensed in dust grains, as in the Galactic interstellar medium, dust cooling triggers re-fragmentation of a clump into sub-solar mass pieces, for metallicities higher than $\sim 10^{-5} \mathrm{Z}_{\odot}$. Therefore, the presence of dust is essential in low-mass $\left(\lesssim \mathrm{M}_{\odot}\right)$ star formation from a shock-compressed cloud.
\end{abstract}

Key words: stars: formation, stars: Population III, stars: Population II

\section{INTRODUCTION}

Theories predict that the first stars formed from primordial pristine gas, the Population III (Pop III) stars, were typically massive with 10-1000 $\mathrm{M}_{\odot}$ (e.g., Bromm et al. 2002; Abel et al. 2002; Yoshida et al. 2008; Hosokawa et al. 2012; Hirano et al. 2014; Susa et al. 2014), while Pop I stars in the solar neighborhood have a peak in their initial mass function (IMF) around 0.1-1 $\mathrm{M}_{\odot}$ (e.g., Kroupa 2002; Bastian et al. 2010). Although the IMF of metal-poor Pop II stars is still uncertain, some of them are surely lowmass objects as discovered in the Galactic halo, globular clusters, and nearby dwarf galaxies (e.g, Beers \& Christlieb 2005; Frebel \& Norris 2015). This indicates a transition from high-mass to low-mass star-formation mode, so-called the Pop III/II transition, over cosmic history. Such a transition might have been caused by the increasing contribution of turbulence, magnetic fields and metals in star formation. Metals are known to affect the thermal properties of a starforming gas by elevating its cooling efficiency and thereby

^ E-mail: nakauchi@astr.tohoku.ac.jp reducing the cloud fragmentation mass scale (e.g., Omukai 2000).

Metals can exist in two forms, i.e., in the gasphase or in dust grains. For metallicities higher than $Z / \mathrm{Z}_{\odot}=10^{-4}-10^{-3}$, fine-structure line cooling by gas-phase metals enables the gas to reach lower temperatures (at a density of $\sim 10^{4} \mathrm{~cm}^{-3}$ ) than in the primordial case, lowering the fragmentation mass (Bromm et al. 2001; Bromm \& Loeb 2003; Santoro \& Shull 2006; Jappsen et al. 2007; Glover \& Clark 2014). This cooling is, however, efficient only at rather low densities $\left(\sim 10^{4} \mathrm{~cm}^{-3}\right)$ and leads to fragments with mass scales no smaller than a few $10 \mathrm{M}_{\odot}$. If some fraction of the metals are in dust grains, as in the Galactic interstellar medium (ISM), dust cooling triggers fragmentation at high enough densities $\left(\gtrsim 10^{10} \mathrm{~cm}^{-3}\right)$ to produce sub-solar mass fragments for metallicities exceeding $Z / \mathrm{Z}_{\odot}=10^{-6}-10^{-5}$ (Schneider et al. 2002, 2003; Omukai et al. 2005; Schneider et al. 2006; Omukai et al. 2010; Schneider \& Omukai 2010; Schneider et al. 2012a,b). Three-dimensional hydrodynamic calculations also demonstrate the formation of multiple low-mass stars through dust-induced fragmentation for $Z / \mathrm{Z}_{\odot} \sim 10^{-5}$ (Tsuribe \& Omukai 2006; Clark et al. 2008; 
Dopcke et al. 2013; Chiaki et al. 2016; Bovino et al. 2016). Therefore, dust is considered to be indispensable to form low-mass $\left(\lesssim \mathrm{M}_{\odot}\right)$ stars.

Shocks are presumably prevalent also in the early galaxies, associated with virialization, galaxy mergers, expansion of HII regions, outflows, SN explosions etc. For example, numerical simulations demonstrate that inflows of cold gas penetrate deep in a forming galaxy and create shocks in a dense region (Wise \& Abel 2007; Wise et al. 2008; Greif et al. 2008; Safranek-Shrader et al. 2014, 2016). The fragmentation mass-scale in such a shock-compressed layer of low metallicity gas was studied by Safranek-Shrader et al. (2010), using a semi-analytical method. They calculated the post-shock thermal evolution starting from the initial density and temperature of $n_{\mathrm{H}, 1}=4 \times 10^{3} \mathrm{~cm}^{-3}$ and $T_{1}=$ $1.1 \times 10^{4} \mathrm{~K}$, suggested by the result of a numerical simulation by Greif et al. (2008). They found that the temperature reaches the CMB floor via metal-line cooling and moderately low-mass fragments $\left(\sim 3 \mathrm{M}_{\odot}\right)$ are formed even without dust, if the metallicity is higher than $Z / \mathrm{Z}_{\odot} \simeq 10^{-2.5}$.

If the star-formation efficiency from the parent dense cores to the stars is about $30 \%$, as suggested by observations in the solar neighborhood, or smaller, this implies the possibility to form solar or sub-solar mass stars even in the absence of dust cooling. Their calculation, however, lacked some important thermal processes, like line cooling by metallic molecules (e.g., $\mathrm{H}_{2} \mathrm{O}, \mathrm{OH}$, and $\mathrm{CO}$ ), cooling by dust thermal emission, and heating associated with $\mathrm{H}_{2}$ formation. The evolution of a self-gravitating clump after fragmentation was not followed in their work either. In addition, a restricted set of physical conditions (initial density, UV strength, and CMB temperature) was investigated, limiting our ability to understand the prevalence of shock-induced low-mass star formation.

Here, by modeling the thermal and chemical processes in more detail, we study thermal evolution and fragmentation of shock-compressed clouds, for a large range of initial density, metallicity, UV strength, and CMB temperature. Our aim is to pin down the condition for low-mass star formation. We find that the mass of a fragment formed by shock-compression does not become smaller than $\simeq 3 \mathrm{M}_{\odot}$ in any environmental conditions we explored. If the metallicity is higher than $\sim 10^{-5} \mathrm{Z}_{\odot}$ and a similar fraction of metals as in the Milky Way condenses into dust grains, dust cooling enables re-fragmentation into sub-solar mass dense cores, irrespective of the initial density and UV strength. Low-mass star formation is possible only in shock-compressed clouds which contain dust grains and have metallicity higher than $\sim 10^{-5} \mathrm{Z} \odot$.

The rest of the paper is organized as follows. In Section 2, we describe the method to calculate the thermal evolution of a shock-compressed cloud leading to fragmentation, as well as the subsequent gravitational contraction of the fragments. Results for thermal evolution are presented in Section 3, where we also discuss their dependences on the UV strength and CMB temperature. We focus on two specific sets of initial conditions that are expected in early galaxies, i.e., cold accretion flows and SN explosions. Fragmentation mass scales are summarized in Section 4. We also discuss the condition for low-mass star formation, by seeing the fragment mass formed under various conditions. Section 5 is devoted to summary and discussion.

\section{METHOD}

\subsection{Post-shock Thermal Evolution}

We calculate the thermal evolution of a shock-compressed layer under the assumption of a plane-parallel and steady flow (Shapiro \& Kang 1987; Yamada \& Nishi 1998; Inayoshi \& Omukai 2012; Nakauchi et al. 2014). The physical quantities are functions only of the distance $x$ from the shock. This treatment corresponds to calculating a spatially averaged profile on the $x=$ const. surface although the postshock medium can be inhomogeneous by thermal instabilities etc.

Density $\rho$, velocity $v$, and pressure $P$ are followed by using the mass and momentum conservations:

$\rho_{1} v_{1}=\rho v$,

and

$\rho_{1} v_{1}^{2}+P_{1}=\rho v^{2}+P$,

where the subscript ' 1 ' indicates the quantities immediately behind the shock front. The internal energy $e$ per unit mass is obtained by solving the energy equation:

$v \frac{d e}{d x}=-P v \frac{d}{d x}\left(\frac{1}{\rho}\right)-\Lambda_{\text {net }}$,

where $\Lambda_{\text {net }}\left(\operatorname{erg~g}^{-1} \mathrm{~s}^{-1}\right)$ is the net cooling rate, which is supplemented by the relations $P=\rho k_{\mathrm{B}} T / \mu m_{\mathrm{H}}$ and $e=$ $P / \rho /(\gamma-1)$. Here, the symbols have their usual meanings.

For the cooling rate, $\Lambda_{\text {net }}$, we consider the following cooling/heating processes: radiative cooling of atomic and molecular lines $\left(\Lambda_{\text {line }}\right)$, and of continuum from gas and dust $\left(\Lambda_{\text {cont }}\right.$ and $\left.\Lambda_{\text {grain }}\right)$, heating/cooling associated with chemical reactions $\left(\Lambda_{\text {chem }}\right)$, and photoelectric heating by dust grains $\left(\Gamma_{\mathrm{pe}}\right)$ :

$\Lambda_{\text {net }}=\Lambda_{\text {line }}+\Lambda_{\text {cont }}+\Lambda_{\text {grain }}+\Lambda_{\text {chem }}-\Gamma_{\text {pe }}$.

Line cooling $\left(\Lambda_{\text {line }}\right)$ is contributed by $\mathrm{H}$ Ly $\alpha$, the rovibrational lines of $\mathrm{H}_{2}, \mathrm{HD}, \mathrm{H}_{2} \mathrm{O}, \mathrm{OH}$, and $\mathrm{CO}$, and the finestructure lines of [OI], [CII], [CI], [SiII], and [FeII]. The cooling and heating rates are calculated following Omukai et al. (2010) for $\mathrm{H}_{2} \mathrm{O}, \mathrm{OH}$, and $\mathrm{CO}$ cooling, Schneider et al. (2012a) for [SiII] and [FeII] cooling, Omukai et al. (2008) for photoelectric heating $\Gamma_{\text {pe }}$, and Omukai et al. (2005) for the remaining processes except for the $[\mathrm{OI}]$ and $[\mathrm{CI}]$ cooling rates, which are updated by using the collisional excitation rates for hydrogen impact computed by Abrahamsson et al. (2007). Reduction of cooling/heating rates due to photon trapping is taken into account by considering the frequency shift due to velocity gradient: the line optical depth is evaluated from the column density across the layers whose velocity difference is less than the thermal velocity.

Following Omukai (2012), we calculate the H, He, D, C and $\mathrm{O}$ chemical network among the 50 species: $\mathrm{H}, \mathrm{H}_{2}, \mathrm{e}^{-}$, $\mathrm{H}^{+}, \mathrm{H}_{2}^{+}, \mathrm{H}_{3}^{+}, \mathrm{H}^{-}, \mathrm{He}, \mathrm{He}^{+}, \mathrm{He}^{2+}, \mathrm{HeH}^{+}, \mathrm{D}, \mathrm{HD}, \mathrm{D}^{+}, \mathrm{HD}^{+}$, $\mathrm{D}^{-}, \mathrm{C}, \mathrm{C}_{2}, \mathrm{CH}, \mathrm{CH}_{2}, \mathrm{CH}_{3}, \mathrm{CH}_{4}, \mathrm{C}^{+}, \mathrm{C}_{2}^{+}, \mathrm{CH}^{+}, \mathrm{CH}_{2}^{+}, \mathrm{CH}_{3}^{+}$, $\mathrm{CH}_{4}^{+}, \mathrm{CH}_{5}^{+}, \mathrm{O}, \mathrm{O}_{2}, \mathrm{OH}, \mathrm{CO}, \mathrm{H}_{2} \mathrm{O}, \mathrm{HCO}, \mathrm{O}_{2} \mathrm{H}, \mathrm{CO}_{2}, \mathrm{H}_{2} \mathrm{CO}$, $\mathrm{H}_{2} \mathrm{O}_{2}, \mathrm{O}^{+}, \mathrm{O}_{2}^{+}, \mathrm{OH}^{+}, \mathrm{CO}^{+}, \mathrm{H}_{2} \mathrm{O}^{+}, \mathrm{HCO}^{+}, \mathrm{O}_{2} \mathrm{H}^{+}, \mathrm{H}_{3} \mathrm{O}^{+}$, $\mathrm{H}_{2} \mathrm{CO}^{+}, \mathrm{HCO}_{2}^{+}$, and $\mathrm{H}_{3} \mathrm{CO}^{+}$. The $\mathrm{Si}$ and $\mathrm{Fe}$ chemistries are not solved, and we assume these elements to be always in the form of SiII and FeII.

All the photo-reaction rates are scaled by a single parameter, the so-called Habing parameter $G_{0}$, defined as the 
energy density between $6-13.6 \mathrm{eV}$, normalized by the Galactic field $5.29 \times 10^{-14} \mathrm{erg} \mathrm{cm}^{-3}$ (Habing 1968). Here, the UV spectrum is implicitly assumed to be the same as the local interstellar field. The Habing parameter $G_{0}$ is related to another conventional UV parameter, $J_{21}$, the intensity at the Lyman limit normalized by $10^{-21} \mathrm{erg} \mathrm{s}^{-1} \mathrm{~cm}^{-2} \mathrm{~Hz}^{-1} \mathrm{str}^{-1}$ as

$J_{21}=20.9 G_{0}$,

for the spectrum of the local interstellar field (Mathis et al. 1983). This relation depends on the spectral shape. For example, $J_{21}=0.667 G_{0}\left(102 G_{0}\right)$ for a black-body spectrum with $10^{4} \mathrm{~K}\left(10^{5} \mathrm{~K}\right)$. Among dozens of photo-reactions included in our calculation, the two most relevant to thermal evolution are $\mathrm{H}_{2}$ photo-dissociation and $\mathrm{C}$ photoionization, both of which are controlled by the radiation in the frequency range $\simeq 11-12 \mathrm{eV}$. If the value of $J_{21}$ is same, the intensity at $\simeq 11 \mathrm{eV}$ for the black body spectrum with $10^{4}$ $\mathrm{K}\left(10^{5} \mathrm{~K}\right)$ is larger by a factor of $3.7(0.27)$ compared to the Mathis et al. (1983) spectrum. Therefore, our results calculated with a certain UV strength of $J_{21}$ reproduce those calculated with $0.27 J_{21}\left(3.7 J_{21}\right)$ for the black body spectrum with $10^{4} \mathrm{~K}\left(10^{5} \mathrm{~K}\right)$.

\subsection{Fragmentation Criterion of a Shock-Compressed Layer}

Here, we consider the condition for fragmentation of the shock-compressed layers, which produces self-gravitating clumps. Elmegreen \& Elmegreen (1978) performed a linear perturbation analysis for a plane-parallel layer in hydrostatic equilibrium and bounded from both sides by external pressure. They find that the most unstable perturbation mode of approximately the layer width grows roughly in the free-fall time scale, $t_{\mathrm{ff}} \equiv \sqrt{3 \pi / 32 G \rho}$, resulting in fragmentation of the layer. In our model, we also need to take the contraction of the layer into account. The steady post-shock flow cools almost isobarically and contracts in the dynamical time scale which is comparable to the cooling time: from the isobaricity and Eq. $3, t_{\mathrm{dyn}} \equiv \rho / \dot{\rho}=\gamma\left(e / \Lambda_{\text {net }}\right)=\gamma t_{\text {cool }} \sim t_{\text {cool }}$. Thus if $t_{\mathrm{cool}}<t_{\mathrm{ff}}$, the layer contracts further before the perturbations grow and no fragmentation occurs. Conversely, if $t_{\text {cool }}>t_{\text {ff }}$, the perturbations have enough time to grow and the shocked layer fragments before further contraction. These fragments are, however, not self-gravitating unless the free-fall time $t_{\mathrm{ff}}$ is shorter than the sound crossing time $t_{\text {sound }}$ across the layer. We thus adopt the following inequalities in time-scales as the fragmentation condition:

$t_{\mathrm{cool}}>t_{\mathrm{ff}}$ and $t_{\mathrm{sound}}>t_{\mathrm{ff}}$

Here, the sound crossing time is evaluated by $t_{\text {sound }}=$ $H_{\rho} / c_{\mathrm{s}}$, using the density scale height of the coolest layer $H_{\rho}=\rho /(d \rho / d r)$ and its sound speed $c_{\mathrm{s}}=(\gamma P / \rho)^{1 / 2}$. Note that Safranek-Shrader et al. (2010) adopted only the second condition $\left(t_{\text {sound }}>t_{\mathrm{ff}}\right)$ as the criterion of fragmentation, which indicates that the layer becomes self-gravitating. The first condition $\left(t_{\mathrm{cool}}>t_{\mathrm{ff}}\right)$ assures that perturbations have enough time to grow.

\subsection{Gravitational Contraction of Clumps after Fragmentation}

After fragmentation, the clumps continue to contract by selfgravity. For spherically symmetric clumps without turbulence or magnetic fields, hydrodynamical studies tell us that the actual contraction follows the Larson-Penston type selfsimilar solution, where the central density increases in the local free-fall time scale (Larson 1969; Penston 1969). We follow its central evolution using a one-zone model:

$\frac{d \rho}{d t}=\frac{\rho}{t_{\mathrm{ff}}}$,

and

$\frac{d e}{d t}=-P \frac{d}{d t}\left(\frac{1}{\rho}\right)-\Lambda_{\text {net }}$,

coupled with the same cooling rate as in Eq. (4). Since the core size is comparable to the local Jeans length, defined by $\lambda_{\mathrm{J}} \equiv\left(\pi k_{\mathrm{B}} T / G \mu m_{\mathrm{H}} \rho\right)^{1 / 2}$, photon trapping and shielding are evaluated by using the column density across the core: $N_{\mathrm{H}, \text { core }}=n_{\mathrm{H}} \lambda_{\mathrm{J}}$.

\subsection{Fragmentation Mass Scale}

The clump mass at its formation by fragmentation can be evaluated from the Jeans mass, $M_{\mathrm{J}}(\rho, T)$, by using the density $\rho_{\text {frag }}$ and temperature $T_{\text {frag }}$ at that epoch:

$M_{\mathrm{J}}\left(\rho_{\text {frag }}, T_{\text {frag }}\right) \simeq \rho_{\text {frag }} \lambda_{\mathrm{J}}^{3}\left(\rho_{\text {frag }}, T_{\text {frag }}\right)$.

For the clump to continue its gravitational contraction, its mass should always exceed the instantaneous Jeans mass $M_{\mathrm{J}}(\rho, T)$. If the temperature increases so rapidly that the effective ratio of specific heat $\gamma_{\text {eff }}(\equiv d \ln P / d \ln \rho)$ exceeds $4 / 3$, the instantaneous Jeans mass increases with contraction and can eventually exceed the clump mass. In such cases, the clump would contract in a quasi-static manner by accreting surrounding material, keeping its mass roughly at the instantaneous Jeans mass. Therefore, the final clump mass is given by the maximum value of the instantaneous Jeans mass $M_{\mathrm{J}}(\rho, T)$ reached during gravitational contraction: $M_{\text {clump }}=\max \left[M_{\mathrm{J}}(\rho, T)\right]$.

Re-fragmentation of a clump can occur during gravitational contraction, if the clump experiences a rapid cooling phase. Following Schneider \& Omukai (2010), we assume that fragmentation occurs if the temperature drops suddenly with $\gamma_{\mathrm{eff}}<0.8$. The fragment mass is set by the Jeans mass when the cooling phase is almost over with $\gamma_{\text {eff }} \geq 0.97$. Such fragmentation can be driven either by line or dust cooling (Schneider et al. 2002; Omukai et al. 2005; Tsuribe \& Omukai 2006, 2008). We call this fragment mass scale as $M_{\text {re-frag. }}$.

\subsection{Initial Settings}

We study the evolution of a shock-compressed cloud, focusing mainly on two situations. The first is a cold accretion (CA) flow penetrating into a protogalaxy with a speed of $\sim 20 \mathrm{~km} \mathrm{~s}^{-1}$. Shocks associated with it heat the medium in the dense region of a protogalaxy, whose typical density is $\sim 10^{3} \mathrm{~cm}^{-3}$ (Wise \& Abel 2007; Wise et al. 2008; Greif et al. 2008; Safranek-Shrader et al. 2010). Therefore, 
in the CA case, the initial density and temperature are set at $n_{\mathrm{H}, 1}=4 \times 10^{3} \mathrm{~cm}^{-3}$ and $T_{1}=1.2 \times 10^{4} \mathrm{~K}$, respectively. The second is a $\mathrm{SN}$ shock propagating into an HII region with $\sim 0.1-1 \mathrm{~cm}^{-3}$, which surrounds the progenitor massive star (Kitayama \& Yoshida 2005; Nagakura et al. 2009; Chiaki et al. 2013). In the early snow-plough phase of a SN remnant (SNR), the temperature of a layer immediately behind the shock front becomes higher than $\sim 10^{5} \mathrm{~K}$ due to the large shock velocities of $\sim 50-100 \mathrm{~km} \mathrm{~s}^{-1}$. It soon, however, decreases down to $\sim 10^{4} \mathrm{~K}$ via $\mathrm{H}$ and $\mathrm{He}$ atomic cooling. In the $\mathrm{SN}$ case, we start the calculation from a post-shock layer that has already compressed to $n_{\mathrm{H}, 1}=4 \mathrm{~cm}^{-3}$ and $T_{1}=1.2 \times 10^{4} \mathrm{~K}$. Considering the possibility that a SN shock sweeps up an ISM denser than $\sim 0.1-1 \mathrm{~cm}^{-3}$, we explore a larger range of initial densities with $1 \mathrm{~cm}^{-3}<n_{\mathrm{H}, 1}<10^{5} \mathrm{~cm}^{-3}$. In the snow plough phase, the width of the post shock layer is much smaller than the radius of a shock front and the curvature of the shock is negligible, as shown by the hydrodynamical calculation of a SNR evolution (Nagakura et al. 2009). Therefore, the planeparallel shock model is also applicable to the SN case.

We study metal-poor clouds in the metallicity range of $0<Z / \mathrm{Z}_{\odot} \leq 10^{-2}$, and set the $\mathrm{CMB}$ temperature at $T_{\mathrm{CMB}}=$ $30 \mathrm{~K}$. The case of a lower CMB temperature, $T_{\mathrm{CMB}}=10 \mathrm{~K}$, is also studied for comparison. The UV strength in early galaxies is highly uncertain. If stars are formed much more actively in the early galaxies than in the solar neighborhood, the UV strength can be much higher than the local interstellar value, $J_{21} \simeq 20\left(G_{0} \simeq 1\right)$ (Mathis et al. 1983). Therefore, we explore a broad range of UV strengths: $0 \leq J_{21} \leq 10^{4}$.

The initial fractions of $\mathrm{H}^{+}, \mathrm{H}_{2}$, and $\mathrm{HD}$ are set as $y\left(\mathrm{H}^{+}\right)_{\mathrm{ini}}=10^{-4}, y\left(\mathrm{H}_{2}\right)_{\mathrm{ini}}=10^{-6}$, and $y(\mathrm{HD})_{\mathrm{ini}}=10^{-9}$, respectively (e.g., Galli \& Palla 2013). The other H and D nuclei are in the neutral atoms. The elemental abundances of $\mathrm{D}$ and He are $y_{\mathrm{D}}=2.5 \times 10^{-5}$ and $y_{\mathrm{He}}=8.3 \times 10^{-2}$, respectively. The amount of metals depleted into dust grains affect the metal abundances in the gas-phase. In principle, the relative abundances of metals and dust should depend on the chemical enrichment history of the galaxy (Schneider et al. 2006, 2012b; Marassi et al. 2015; de Bennassuti et al. 2014, 2017; Ginolfi et al. 2018). However, here we ignore this complication and simply assume that, when no dust is present, the gas-phase metal composition is proportional to the solar abundance pattern (Anders \& Grevesse 1989). On the other hand, when dust is present, we take the gas-phase metal composition and dust-to-gas ratio to follow the values of local ISM, i.e, 72, 46, 90, and $100 \%$ of C, O, Si, and Fe are depleted into dust grains as modeled by Pollack et al. (1994). Since the initial temperature is $\simeq 10^{4} \mathrm{~K}$, all the He, $\mathrm{C}$ and $\mathrm{O}$ are supposed to be in HeI, CII and OI.

The initial abundances of $\mathrm{H}^{+}, \mathrm{H}_{2}$ and $\mathrm{HD}$, adopted in our calculation, are the values expected in the highredshift intergalactic medium, and may not be appropriate for a $\mathrm{CA}$ and a $\mathrm{SN}$ shock. Without an external ionization source, the recombination proceeds in a free-fall time to $y\left(\mathrm{H}^{+}\right) \sim\left(10^{-4}-10^{-3}\right) \times n_{\mathrm{H}}^{-1 / 2}$ in a warm neutral medium of several $1000 \mathrm{~K}$, even with a higher ionization fraction initially. Although this value is more or less similar to our adopted abundance for the relatively diffuse case with $n_{\mathrm{H}} \sim 10 \mathrm{~cm}^{-3}$, the initial ionization fraction can differ in the cases with a largely different initial density. However, we believe our results to be robust due to the following consid- erations. Even with a smaller initial ionization degree, the high initial temperature of $\gtrsim 10000 \mathrm{~K}$ leads to a rapid increase of the ionization fraction to $y\left(\mathrm{H}^{+}\right) \sim 4 \times 10^{-4}$ via collisional ionization, erasing the memory of the initial low ionization degree. A higher value of ionization degree also has only a minor effect. The ionization fraction decreases by recombination to a similar level as in the low $y\left(\mathrm{H}^{+}\right)_{\text {ini }}$ cases during the isobaric contraction stage, whose track is solely determined by the shock velocity. Thus, different ionization degrees do not largely alter our thermal tracks. In addition, $\mathrm{H}_{2}$ and $\mathrm{HD}$ initially present are collisionally dissociated due to the high initial temperature $(\sim 10000 \mathrm{~K})$ and their abundances decrease rapidly to $y\left(\mathrm{H}_{2}\right) \sim 10^{-7}$ and $y(\mathrm{HD}) \sim 10^{-11}$, respectively. By the time the gas cools to $\sim$ $1000 \mathrm{~K}, \mathrm{H}_{2}$ and $\mathrm{HD}$ are formed again with their abundances reaching $y\left(\mathrm{H}_{2}\right) \sim 10^{-3}$ and $y(\mathrm{HD}) \sim 10^{-7}$, respectively. Therefore, $y\left(\mathrm{H}_{2}\right)$ and $y(\mathrm{HD})$ values in the subsequent stages do not depend on their initial values.

\section{THERMAL EVOLUTION}

In this section, we describe the thermal evolution of a shockcompressed cloud. In Sections 3.1 and 3.2, we present the results for $\mathrm{CA}$ and $\mathrm{SN}$ shocks, respectively, in the case of no UV irradiation $\left(J_{21}=0\right)$. In Sections 3.3 and 3.4, we see how the results are altered by the presence of UV irradiation and by a lower CMB temperature, respectively.

\subsection{Cloud Compressed by a Cold Accretion Shock}

Thermal evolution of clouds compressed by CA shocks in the absence of UV irradiation $\left(J_{21}=0\right)$ is shown in Fig. 1, for initial metallicities of (a) $Z / \mathrm{Z}_{\odot}=0$, (b) $10^{-4}$, (c) $10^{-3}$, and (d) $10^{-2}$. The blue and red lines in each panel show the cases with and without dust, respectively. The circles and triangles on the thermal tracks indicate the epochs of clump formation and its re-fragmentation, respectively. The black-dashed lines show the thermal tracks of initially ionized clouds ('pre-ionized' clouds, hereafter) that start their collapse without shock-compression, but with high ionization degree and temperature, similar to a shock-compressed gas (Omukai 2012). They are presented for comparison to see how thermal tracks differ between a gas with and without shock-compression. For the pre-ionized clouds, calculation is started from $n_{\mathrm{H}}=1.0 \mathrm{~cm}^{-3}, T=10^{4} \mathrm{~K}$, and $y(e)=0.1$ and the remaining conditions are the same as those of shocked clouds at each metallicity. Shaded regions are temperatures below the $\mathrm{CMB}$ value, $T_{\mathrm{CMB}}=30 \mathrm{~K}$, and the diagonal dotted lines show lines of constant Jeans masses.

First, we see the case of zero-metallicity gas (Fig. 1a). The isobaric contraction from the initial temperature and density of $\left(T_{1}, n_{\mathrm{H}, 1}\right)=\left(1.2 \times 10^{4} \mathrm{~K}, 4 \times 10^{3} \mathrm{~cm}^{-3}\right)$ soon terminates at $\left(T, n_{\mathrm{H}}\right)=\left(8000 \mathrm{~K}, 10^{4} \mathrm{~cm}^{-3}\right)$ since Ly $\alpha$ cooling has a cutoff at $8000 \mathrm{~K}$ and the $\mathrm{H}_{2}$ formation is suppressed via collisional dissociation due to the high temperature and density (Inayoshi \& Omukai 2012). At this moment, very massive clumps of $\sim 10^{5}-10^{6} \mathrm{M}_{\odot}$ form (open red circle). Subsequently, the clumps continue collapsing almost isothermally solely via Ly $\alpha$ cooling, with $\mathrm{H}_{2}$ cooling being suppressed. The clumps do not fragment again and supermassive stars 
(a) $C A, Z=0, J_{21}=0$

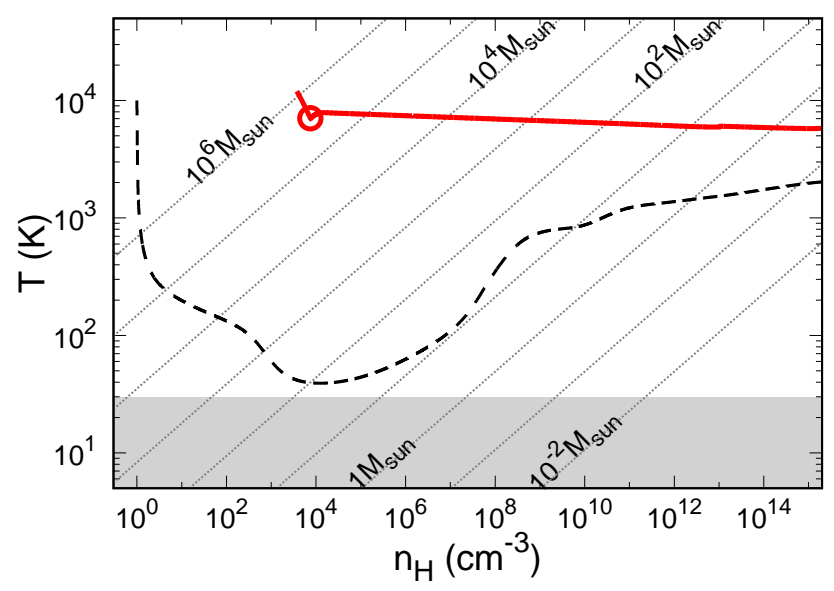

(c) $\mathrm{CA}, 10^{-3} \mathrm{Z}_{\text {sun }}, \mathrm{J}_{21}=0$

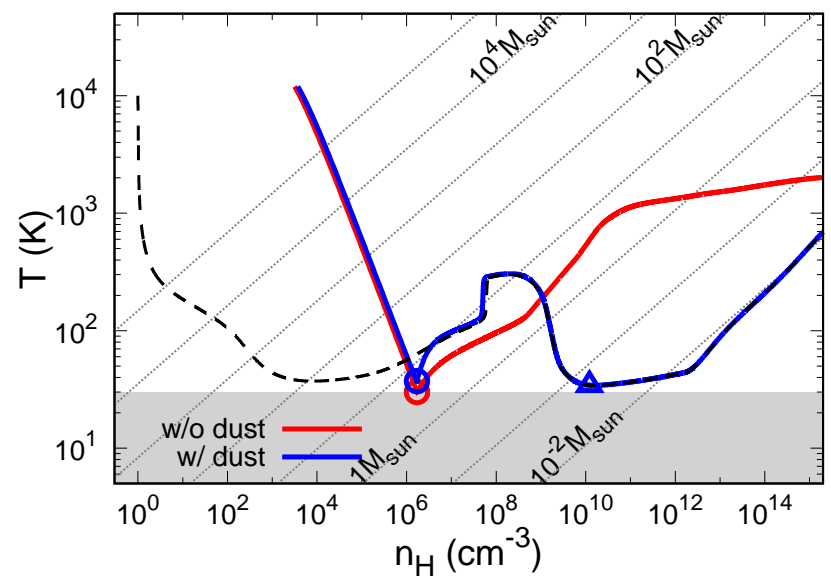

(b) $\mathrm{CA}, 10^{-4} \mathrm{Z}_{\text {sun }}, \mathrm{J}_{21}=0$

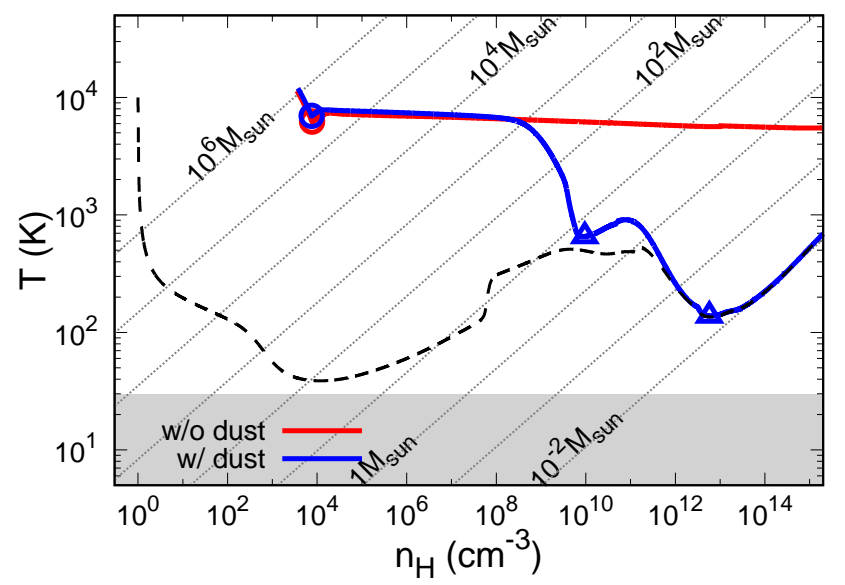

(d) $\mathrm{CA}, 10^{-2} \mathrm{Z}_{\text {sun }}, \mathrm{J}_{21}=0$

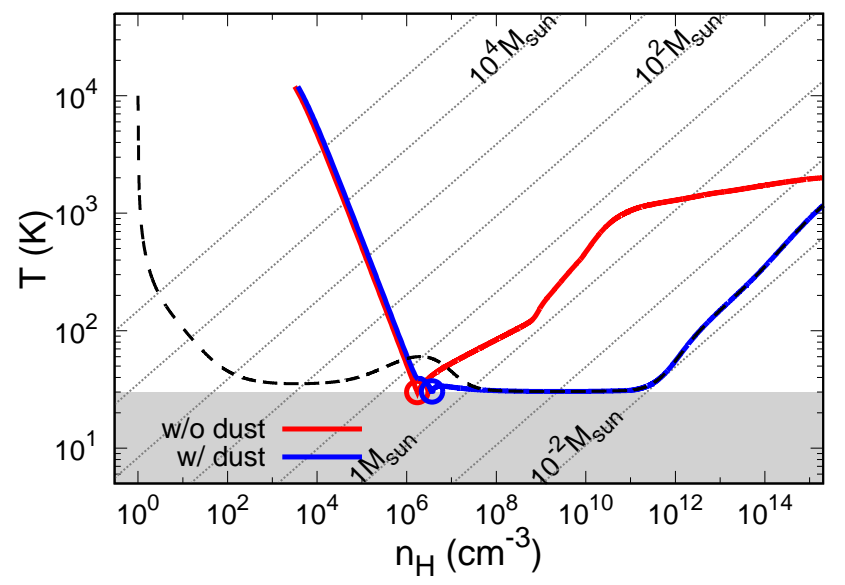

Figure 1. Thermal evolution of a cloud compressed by a CA shock. Panels (a), (b), (c), and (d) correspond to the cases with initial metallicities of $Z / \mathrm{Z}_{\odot}=0,10^{-4}, 10^{-3}$, and $10^{-2}$. The gas is characterized by an initial temperature of $T_{1}=1.2 \times 10^{4} \mathrm{~K}$ and density of $n_{\mathrm{H}, 1}=4 \times 10^{3} \mathrm{~cm}^{-3}$. No UV irradiation is considered $\left(J_{21}=0\right)$. In each panel, solid blue and red lines are the thermal tracks of clouds with and without dust, respectively, and open circles and triangles correspond to the epochs of clump formation and its refragmentation. The black-dashed lines are those of a pre-ionized cloud collapsing by self-gravity, not by shock compression, with the same initial temperature, metallicity, and dust content. The gray-shaded regions are temperatures below the CMB floor that is set at $T_{\mathrm{CMB}}=30 \mathrm{~K}$. Diagonal dotted lines are the loci of constant Jeans mass.

of $\sim 10^{5}-10^{6} \mathrm{M}_{\odot}$ are expected to form (e.g., Nakauchi et al. 2017), eventually leading to a black hole of comparable mass (Inayoshi et al. 2014; Sugimura et al. 2017).

The effects of increasing the gas metallicity are shown in the other panels of Fig. 1. To interpret the evolutionary tracks, it is helpful to look at the relative importance of different cooling/heating processes, which are shown in Fig. 2 for the $10^{-4} \mathrm{Z}_{\odot}$ and $10^{-2} \mathrm{Z}_{\odot}$ models, with and without dust.

We first discuss the models without dust (red solid lines in Fig. 1). The thermal track of $Z=10^{-4} \mathrm{Z}_{\odot}$ (Fig. 1b) is identical to the $Z=0$ track since Ly $\alpha$ cooling is more effective than metal-line cooling (Fig. 2a). The situation changes when the metallicity exceeds $Z \sim 10^{-3} \mathrm{Z}_{\odot}$ (Fig. 1c, d). The isobaric temperature drop continues well below $8000 \mathrm{~K}$ via metal-line cooling, until reaching the CMB floor. $\mathrm{H}_{2}\left(\mathrm{H}_{2} \mathrm{O}\right)$ temporarily becomes the main coolant at $\simeq 1000 \mathrm{~K}$ (below $\simeq 50 \mathrm{~K}$, respectively, see Fig. 2c). Being unable to cool below the CMB temperature, the fragmentation condition (Eq. 6) is met at this stage, and clumps of $\simeq 10 \mathrm{M}_{\odot}$ form (open red circles). During the subsequent collapse of the clumps, the temperature increases rapidly both in $Z=10^{-3}$ and $10^{-2} \mathrm{Z}_{\odot}$ cases, as radiative cooling, mostly by $\mathrm{H}_{2} \mathrm{O}$, cannot catch up with increasing compressional heating. When the density reaches $n_{\mathrm{H}} \sim 10^{9}-10^{10} \mathrm{~cm}^{-3}, \mathrm{H}_{2}$ formation via three-body reactions quickly proceeds and the associated heating balances $\mathrm{H}_{2} \mathrm{O}$ cooling. By $n_{\mathrm{H}} \sim 10^{11} \mathrm{~cm}^{-3}$, all the hydrogen becomes molecular and $\mathrm{H}_{2}$ formation heating declines thereafter. Almost simultaneously, $\mathrm{H}_{2}$ cooling becomes dominant, leading to a shallower temperature increase. When $Z=10^{-3}$ and $10^{-2} \mathrm{Z}_{\odot}$, the clump mass at the end of the isobaric evolution is always larger than the instantaneous Jeans mass during the collapse phase and no fur- 
(a) $C A, 10^{-4} Z_{\text {sun }}$, w/o dust, $J_{21}=0$

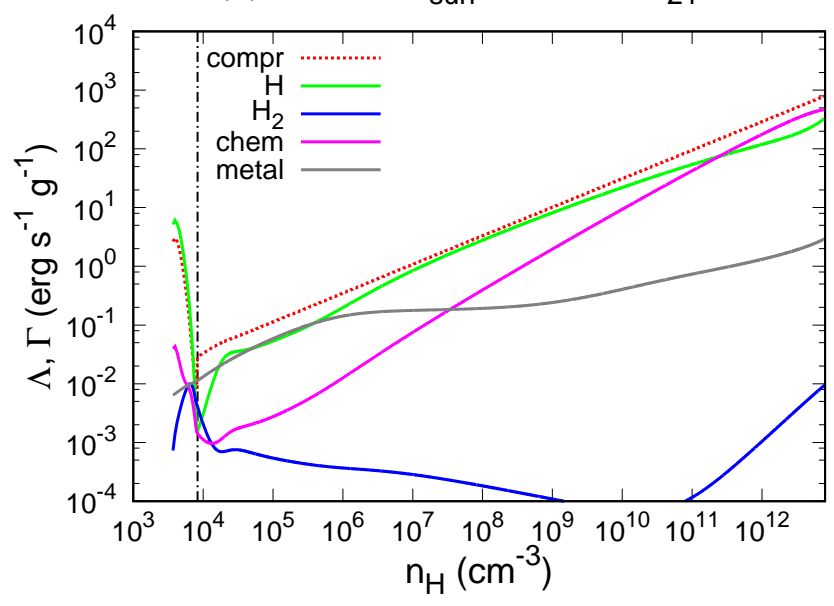

(c) $C A, 10^{-2} Z_{\text {sun }}$, w/o dust, $J_{21}=0$

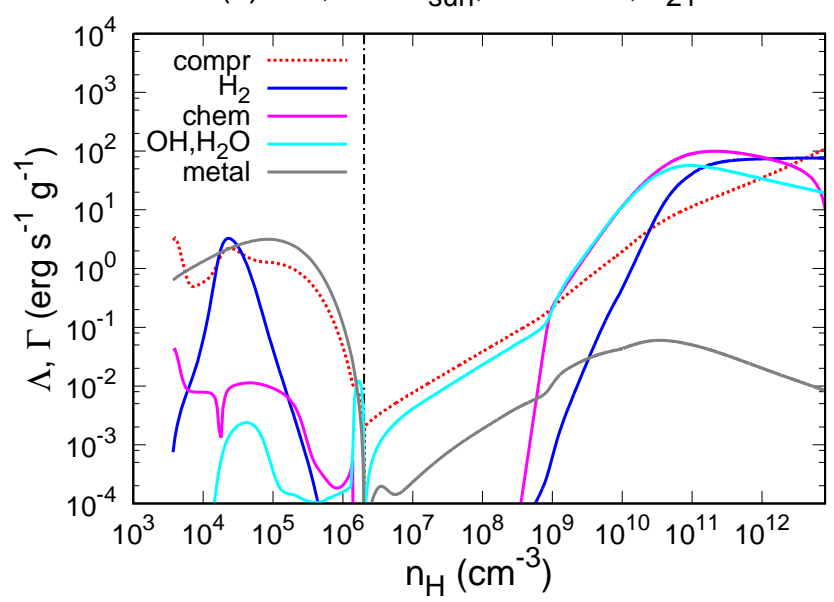

(b) $\mathrm{CA}, 10^{-4} \mathrm{Z}_{\text {sun }}$, w/ dust, $\mathrm{J}_{21}=0$

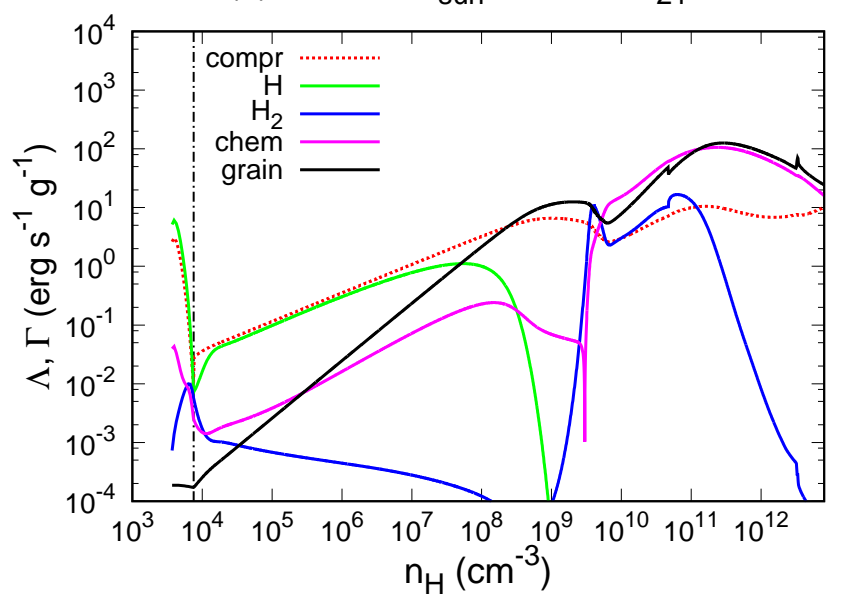

(d) $C A, 10^{-2} Z_{\text {sun }}$, w/ dust, $J_{21}=0$

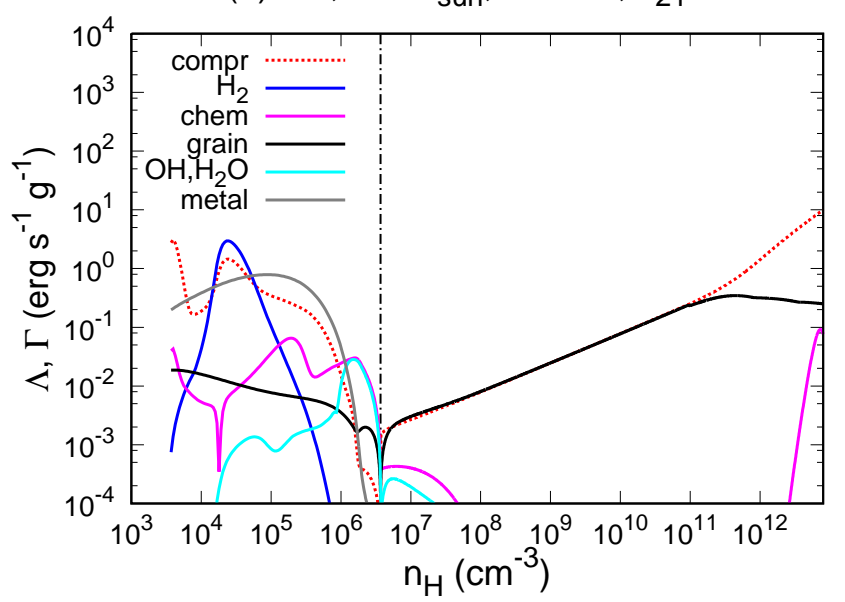

Figure 2. Cooling and heating rates in a cloud compressed by a CA shock. Panels (a), (b), (c), and (d) show the models of $\left(10^{-4} \mathrm{Z}_{\odot}\right.$, without dust), $\left(10^{-4} \mathrm{Z}_{\odot}\right.$, with dust), $\left(10^{-2} \mathrm{Z}_{\odot}\right.$, without dust), and $\left(10^{-2} \mathrm{Z}_{\odot}\right.$, with dust), respectively. Individual cooling/heating processes are indicated in the legend. The vertical dot-dashed line indicates the epoch of clump formation.

ther fragmentation occurs. Therefore, the final clump mass is $M_{\text {clump }} \simeq 10 \mathrm{M}_{\odot}$.

Finally, we discuss the effects of dust (blue solid lines in Fig. 1 and Fig. 2b, d). Dust affects the thermal evolution by means of (i) cooling by thermal emission, (ii) $\mathrm{H}_{2}$ formation on its surface, and when UV radiation is present, (iii) heating by photoelectric emission. The presence of dust has only a minor effect in the isobaric phase, although $\mathrm{H}_{2}$ formation on grain surfaces and the associated heating become effective for the $Z / \mathrm{Z}_{\odot}=10^{-3}$ and $10^{-2}$ cases. When $Z=10^{-2} \mathrm{Z}_{\odot}, \mathrm{H}_{2}$ formation heating creates a small bump in the thermal track at $T \simeq 40 \mathrm{~K}$, which makes the density at fragmentation slightly higher and thus the clump mass slightly smaller $\left(\lesssim 3 \mathrm{M}_{\odot}\right)$ compared to the models without dust. In contrast, dust grains are an important coolant via the thermal emission in the subsequent collapse phase, leading to a large difference between the thermal tracks with and without dust (blue and red lines in Fig. 1b-d). Even when metallicity is as low as $Z=10^{-4} \mathrm{Z}_{\odot}$ (Figs. $1 \mathrm{~b}$ and $2 \mathrm{~b}$ ), after the isothermal contraction at $\sim 8000 \mathrm{~K}$, the tem- perature suddenly drops to $\sim 800 \mathrm{~K}$ at $n_{\mathrm{H}} \sim 10^{9} \mathrm{~cm}^{-3}$ due to dust cooling. Following the short interval of slight temperature increase at $n_{\mathrm{H}} \sim 10^{10}-10^{11} \mathrm{~cm}^{-3}$ caused by $\mathrm{H}_{2}$ formation heating, the temperature further drops to $\sim 100 \mathrm{~K}$ due to dust cooling when $\mathrm{H}_{2}$ formation is almost over. Two open triangles at the local temperature minima indicate the two epochs of re-fragmentation. The second fragmentation episode at $\sim 10^{13} \mathrm{~cm}^{-3}$ produces very low-mass fragments of $M_{\mathrm{re}-\text { frag }} \sim 10^{-2} \mathrm{M}_{\odot}$. When $Z=10^{-3} \mathrm{Z}_{\odot}$ (Fig. 1c), after clump formation at $\sim 10^{6} \mathrm{~cm}^{-3}$, the temperature is raised first gradually and then abruptly at $\sim 10^{8} \mathrm{~cm}^{-3}$ by $\mathrm{H}_{2}$ formation heating via grain surface reactions. The rapid heating at $\sim 10^{8} \mathrm{~cm}^{-3}$ is caused by the increase in the fraction of $\mathrm{H}_{2}$ formation energy released as heat at this density. When $\mathrm{H}_{2}$ formation is almost completed, the temperature drops suddenly to the CMB floor by dust cooling at $\sim 10^{10} \mathrm{~cm}^{-3}$, resulting in the formation of sub-solar mass $\left(M_{\text {re-frag }} \simeq 0.1 \mathrm{M}_{\odot}\right)$ fragments (open triangle). When $Z=10^{-2} \mathrm{Z}_{\odot}$ (Figs. 1d and 2d), a clump formed at the end of the isobaric phase (at $\sim 3 \times 10^{6} \mathrm{~cm}^{-3}$ ) collapses isothermally 
along the CMB floor thanks to efficient dust cooling, and no further fragmentation occurs. Therefore, the clump mass does not change from the initial value $\left(M_{\text {clump }} \lesssim 3 \mathrm{M}_{\odot}\right)$. In all the models with dust, the clouds become optically thick to dust thermal emission at $n_{\mathrm{H}} \sim 10^{12}-10^{13} \mathrm{~cm}^{-3}$ and contract adiabatically thereafter.

Black-dashed lines in Fig. 1 show that the pre-ionized clouds collapsing by self-gravity initially follow a very different evolution compared to the shock-compressed clouds. The temperature is first lowered isochorically until $\simeq 200 \mathrm{~K}$ via efficient $\mathrm{H}_{2}$ cooling, and then is pushed down to the CMB value at $n_{\mathrm{H}} \sim 10^{3}-10^{4} \mathrm{~cm}^{-3}$, via HD cooling for $Z \lesssim 10^{-4} \mathrm{Z}_{\odot}$ and metal-line cooling for $Z \gtrsim 10^{-3} \mathrm{Z}_{\odot}$. After the temperature minima, $\mathrm{H}_{2}$ formation heating via grain surface reactions increases the temperature, until $\mathrm{H}_{2}$ formation is almost completed and dust cooling becomes effective, and the thermal tracks eventually converge to those of the shock-compressed cases. When $Z \gtrsim 10^{-3} \mathrm{Z}_{\odot}$ (Fig. 1c, d), this occurs at a density of $n_{\mathrm{H}} \sim 10^{7}-10^{8} \mathrm{~cm}^{-3}$, which is close to the epoch of clump formation in a shock-compressed cloud. When $Z=10^{-4} \mathrm{Z}_{\odot}$ (Fig. 1b), the thermal tracks converge only after the temperature of a clump is lowered via dust cooling from $8000 \mathrm{~K}$ to $\sim 300 \mathrm{~K}$ at $n_{\mathrm{H}} \sim 10^{12} \mathrm{~cm}^{-3}$.

\subsection{Cloud Compressed by a Supernova Shock}

The thermal evolution and the corresponding cooling/heating rates for a cloud compressed by a SN shock are shown in Figs. 3 and 4, respectively. The main difference from the results of the $\mathrm{CA}$ shock is that the temperature reaches the $\mathrm{CMB}$ value at the end of the isobaric evolution in all the models regardless of metallicity or presence of dust. This is because when the initial density is $n_{\mathrm{H}, 1} \leq 10^{3} \mathrm{~cm}^{-3}$, as in the $\mathrm{SN}$ case, $\mathrm{H}_{2}$ collisional dissociation is inefficient, and the gas can first cool well below $8000 \mathrm{~K}$ via $\mathrm{H}_{2}$ cooling. When $Z \leq 10^{-4} \mathrm{Z}_{\odot}$, HD cooling takes over below 150 $\mathrm{K}$ until the temperature hits the CMB floor (Fig. 4a, b). Metals also become important coolants during the isobaric contraction when $Z \geq 10^{-3} \mathrm{Z}_{\odot}$ (Fig. $4 \mathrm{c}, \mathrm{d}$ ). At $T \sim T_{\mathrm{CMB}}$, the fragmentation condition (Eq. 6) is satisfied and clumps of $\sim 100 \mathrm{M}_{\odot}$ are formed in all the models (open circles in Fig. 3).

The subsequent clump evolution depends strongly on whether dust is present or not. Without dust (solid red lines in Fig. 3), the temperature continues to increase first by compressional heating $\left(\lesssim 10^{8} \mathrm{~cm}^{-3}\right)$ and then by three-body $\mathrm{H}_{2}$ formation heating at higher densities (Fig. 4a, c). The main cooling channels are provided by molecular lines of $\mathrm{H}_{2}$, $\mathrm{HD}, \mathrm{OH}$ and $\mathrm{H}_{2} \mathrm{O}$ and for $Z=10^{-2} \mathrm{Z}_{\odot}$ also by fine-structure lines, but they fail to trigger another phase of rapid cooling and fragmentation. The final clump mass is thus set by the value at the end of the isobaric phase as $M_{\text {clump }} \simeq 100 \mathrm{M}_{\odot}$. In the presence of dust grains (solid blue lines in Fig. 3) the thermal evolution after clump formation at $\sim 10^{3} \mathrm{~cm}^{-3}$ becomes very similar to the pre-ionized cases (black-dashed lines), despite the very different behaviors below that density. During the collapse, the temperature is raised by $\mathrm{H}_{2}$ formation heating via grain surface reactions, and is then lowered rapidly to the local minima through dust cooling at $n_{\mathrm{H}} \sim 10^{8}-10^{12} \mathrm{~cm}^{-3}$ (Fig. 4b, d), where sub-solar mass fragments $\left(M_{\mathrm{re}-\text { frag }} \lesssim 1 \mathrm{M}_{\odot}\right)$ are formed by re-fragmentation of the clumps (open triangles in Fig. 3).

\subsection{Effect of UV Irradiation}

Here, we discuss the effects of UV irradiation on the thermal evolution and fragmentation, by comparing the results obtained assuming $J_{21}=10^{4}$ (solid lines) and $J_{21}=0$ (dashed lines) in Fig. 5. Individual panels refer to the CA models with $10^{-4} \mathrm{Z}_{\odot}$ and $10^{-2} \mathrm{Z}_{\odot}$ (panels a and $\mathrm{c}$ ) and to the SN models with $10^{-4} \mathrm{Z}_{\odot}$ and $10^{-2} \mathrm{Z}_{\odot}$ (panels $\mathrm{b}$ and $\mathrm{d}$ ). Open circles indicate the epochs of clump formation, and asterisks and open triangles indicate those of re-fragmentation caused by line and dust cooling, respectively.

UV irradiation has little impacts on the thermal path in the case of a CA shock (Fig. 5a, c). With the high initial density of $n_{\mathrm{H}, 1}=4 \times 10^{3} \mathrm{~cm}^{-3}, \mathrm{H}_{2}$ cooling is quenched by collisional dissociation at a temperature higher than $\sim 8000 \mathrm{~K}$, independently of the presence of UV irradiation. When $Z=$ $10^{-4} \mathrm{Z}_{\odot}$ (Fig. 5a), a very massive clump $\left(\sim 10^{5}-10^{6} \mathrm{M}_{\odot}\right)$ forms at $\left(n_{\mathrm{H}}, T\right) \sim\left(10^{4} \mathrm{~cm}^{-3}, 8000 \mathrm{~K}\right)$ (open circles), as a result of the isobaric evolution via Ly $\alpha$ cooling. This clump follows the same evolutionary track of the $J_{21}=0$ model discussed in Section 3.1. With more metals $\left(Z \gtrsim 10^{-3} \mathrm{Z}_{\odot}\right.$, e.g., Fig. 5c), efficient metal line-cooling decreases the gas temperature below $100 \mathrm{~K}$ in the isobaric phase independently of the UV strength. In the model without dust (red line), a clump is formed at $\left(n_{\mathrm{H}}, T\right) \sim\left(10^{6} \mathrm{~cm}^{-3}, T_{\mathrm{CMB}}\right)$ (open red circle). Since $\mathrm{H}_{2} \mathrm{O}$ is not shielded against UV in our model, $\mathrm{H}_{2} \mathrm{O}$ formation remains suppressed via photodissociation for $n_{\mathrm{H}} \sim 10^{6}-10^{10} \mathrm{~cm}^{-3}$ and the clump collapses with a slightly higher temperature compared to the $J_{21}=0$ case. At $n_{\mathrm{H}}>10^{10} \mathrm{~cm}^{-3}, \mathrm{H}_{2} \mathrm{O}$ formation overcomes photodissociation and the clump follows the same path as the $J_{21}=0$ case. In the model with dust (blue line), photoelectric heating cannot be neglected at $n_{\mathrm{H}} \sim 10^{5}-10^{6} \mathrm{~cm}^{-3}$, where UV is still not shielded effectively by dust absorption, and the cooling rate becomes less efficient, increasing the cooling time. As a result, the gas satisfies the fragmentation condition (Eq. 6) at a temperature $(\sim 100 \mathrm{~K}$; open blue circle) higher than in the $J_{21}=0$ case, which also makes the clump mass $\left(M_{\text {clump }} \simeq 20 \mathrm{M}_{\odot}\right)$ several times larger. Soon after clump formation, the temperature drops to the $\mathrm{CMB}$ value via dust thermal emission and the clump experiences another episode of fragmentation into solarmass $\left(M_{\mathrm{re}-\text { frag }} \simeq 1 \mathrm{M}_{\odot}\right)$ pieces (open triangle). Although this re-fragmentation is lacking in the $J_{21}=0$ model, the evolutionary tracks of models with $J_{21}=0$ and $10^{4}$ converge thereafter.

A cloud compressed by a SN shock is far more vulnerable to UV irradiation, owing to the lower initial density and weaker UV shielding (Fig. 5b, d). When $\mathrm{H}_{2}$ cooling is strongly suppressed by photodissoiciation, the gas can cool isobarically only up to $8000 \mathrm{~K}$ via Ly $\alpha$ cooling, for most of the models. In these cases, the clump mass becomes very large $M_{\text {clump }} \sim 10^{7} \mathrm{M}_{\odot}$, due to the very low density $\sim 10 \mathrm{~cm}^{-3}$ and high temperature of fragmentation (open circles). When $Z=10^{-4} \mathrm{Z}_{\odot}$ (Fig. 5b), the evolutionary tracks after clump formation are almost the same as in the CA case (Fig. 5a). In the model with dust and with $Z=10^{-2} \mathrm{Z}_{\odot}($ the blue line in Fig. $5 \mathrm{~d}$ ), the temperature in a collapsing clump drops isochorically at $n_{\mathrm{H}} \sim 10^{2} \mathrm{~cm}^{-3}$ via metal-line cooling. When the temperature reaches $\sim 60 \mathrm{~K}$, metal-line cooling becomes inefficient and the clump fragments into massive cores of $M_{\mathrm{re}-\text { frag }} \sim 300 \mathrm{M}_{\odot}$ (asterisk). 
(a) $\mathrm{SN}, \mathrm{Z}=0, \mathrm{~J}_{21}=0$

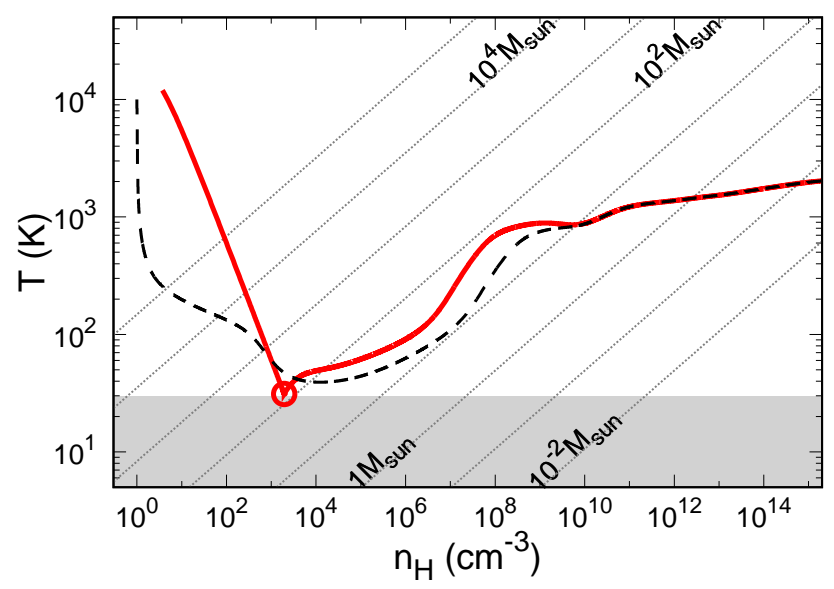

(c) $\mathrm{SN}, 10^{-3} \mathrm{Z}_{\text {sun }}, \mathrm{J}_{21}=0$

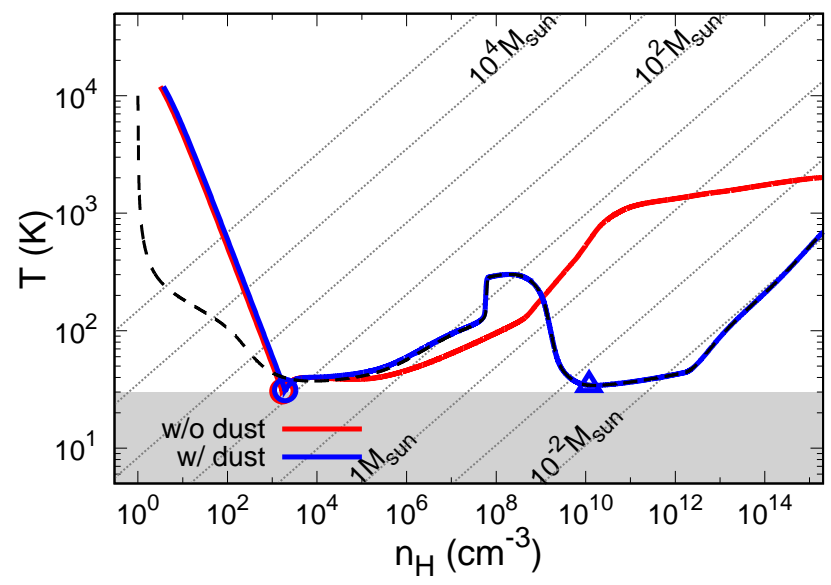

(b) $\mathrm{SN}, 10^{-4} \mathrm{Z}_{\text {sun }}, \mathrm{J}_{21}=0$

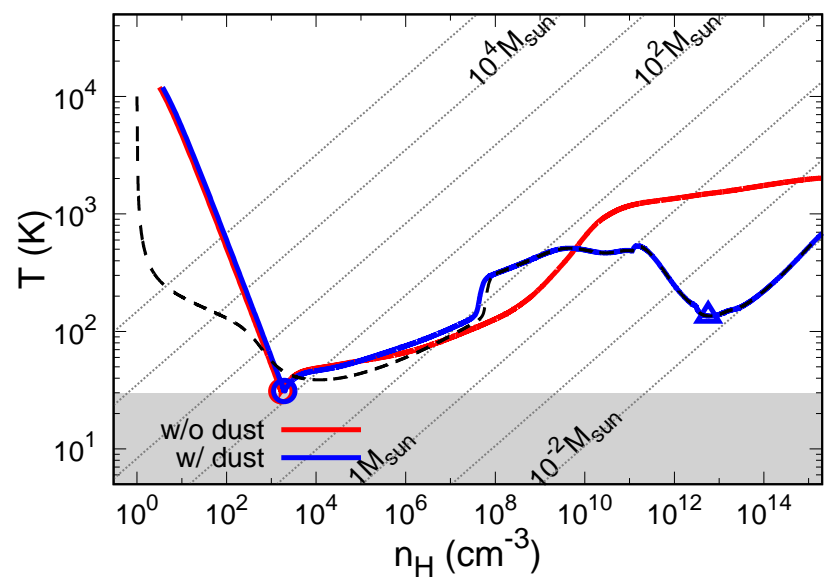

(d) $\mathrm{SN}, 10^{-2} \mathrm{Z}_{\text {sun }}, \mathrm{J}_{21}=0$

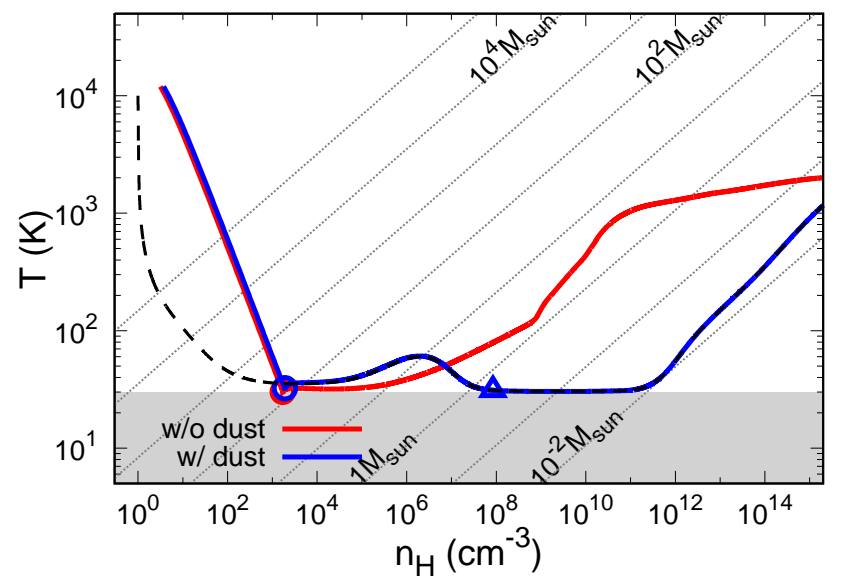

Figure 3. Same as Fig. 1, but for a cloud compressed by a SN shock, which is characterized by the initial density of $n_{\mathrm{H}, 1}=4 \mathrm{~cm}{ }^{-3}$.

These fragments then contract almost isothermally until $n_{\mathrm{H}} \sim 10^{6} \mathrm{~cm}^{-3}$, where the temperature again starts decreasing via dust cooling. The temperature is lowered to the $\mathrm{CMB}$ value at $n_{\mathrm{H}} \sim 10^{8} \mathrm{~cm}^{-3}$, where further fragmentation into much smaller pieces of $M_{\mathrm{re}-\mathrm{frag}} \simeq 1 \mathrm{M}_{\odot}$ occurs (open triangle). Thereafter, the evolutionary track converges on the $J_{21}=0$ case. Note that in the model without dust and with $Z=10^{-2} \mathrm{Z}_{\odot}$ (the red line in Fig. 5d), the temperature reaches the $\mathrm{CMB}$ value during the isobaric evolution, even in the $J_{21}=10^{4}$ model. This is because, for a fixed metallicity, a model without dust contains a larger amount of metals in the gas-phase and efficient metal-line cooling decreases the temperature below $8000 \mathrm{~K}$. During the collapse, the thermal track becomes identical with that in the CA case at $n_{\mathrm{H}}>10^{7} \mathrm{~cm}^{-3}$.

\subsection{Effect of CMB}

The CMB affects the low-metallicity gas evolution and fragmentation by setting a minimum temperature floor that gas can reach, as studied in Schneider \& Omukai (2010) by a semi-analytical approach and in Smith et al. (2009) by three-dimensional hydrodynamic calculations, although the latter does not take dust cooling and $\mathrm{H}_{2}$ formation heating into account. Here, we see the CMB effects by changing the CMB temperature to $T_{\mathrm{CMB}}=10 \mathrm{~K}$. In Fig. 6 , we show the thermal tracks with $T_{\mathrm{CMB}}=10 \mathrm{~K}$ (solid lines) for the CA models with $10^{-4} \mathrm{Z}_{\odot}$ and $10^{-2} \mathrm{Z}_{\odot}$ (panels a and c) and the SN models with $10^{-4} \mathrm{Z}_{\odot}$ and $10^{-2} \mathrm{Z}_{\odot}$ (panels b and d). The models with $T_{\mathrm{CMB}}=30 \mathrm{~K}$ are also shown for comparison by dashed lines.

When efficient coolants are available, a shocked layer cools isobarically until the temperature hits the CMB floor at $10 \mathrm{~K}$, where clumps are formed by fragmentation. This is the case when $Z \gtrsim 10^{-3} \mathrm{Z}_{\odot}$ in $\mathrm{CA}$ shocks, and the clump mass is $\sim 0.3 \mathrm{M}_{\odot}$ both in models with and without dust (Fig. 6a, c). In SN shocks, the gas reaches the CMB temperature floor at $10 \mathrm{~K}$ in all the models shown in Fig. $6 \mathrm{~b}, \mathrm{~d}$ and clumps with masses $\sim 10-30 \mathrm{M}_{\odot}$ form. Thanks to the lower value of $T_{\mathrm{CMB}}$, the clump mass is one order of magnitude smaller than the $T_{\mathrm{CMB}}=30 \mathrm{~K}$ model. Note that the clump mass at the end of the isobaric evolution can be 
(a) $\mathrm{SN}, 10^{-4} \mathrm{Z}_{\text {sun }}$, w/o dust, $\mathrm{J}_{21}=0$

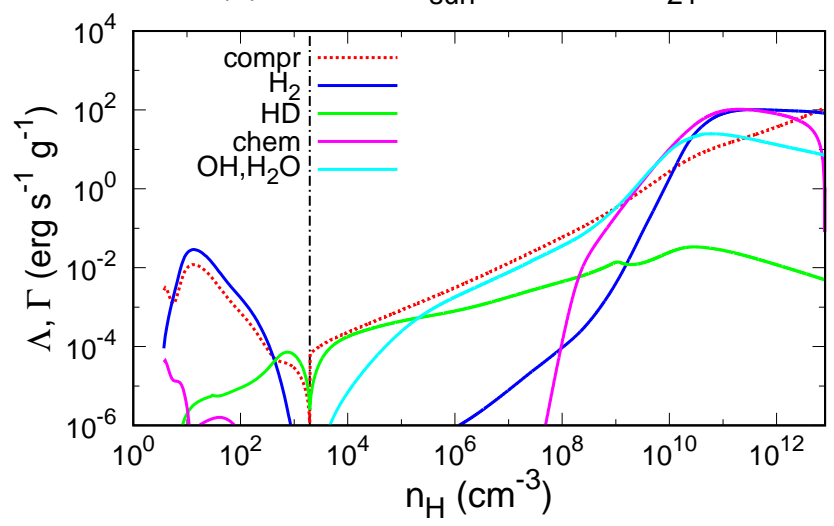

(c) $S N, 10^{-2} Z_{\text {sun }}$, w/o dust, $J_{21}=0$

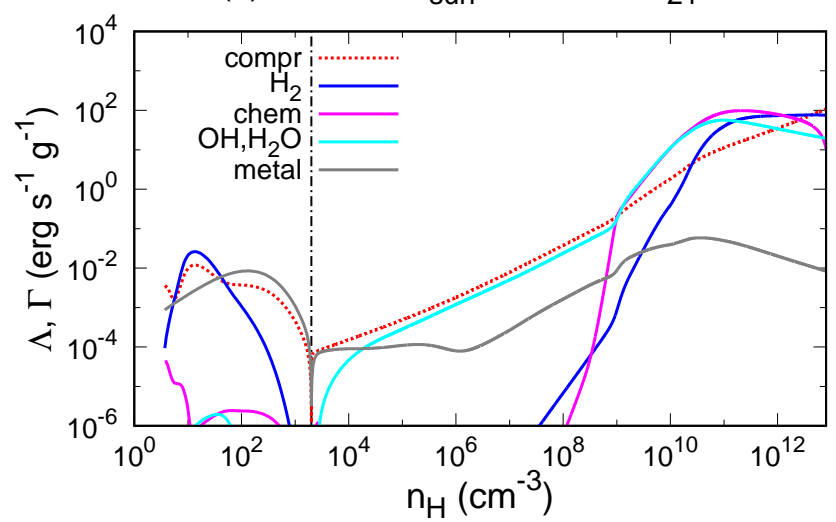

(b) $\mathrm{SN}, 10^{-4} \mathrm{Z}_{\text {sun }}$, w/ dust, $\mathrm{J}_{21}=0$

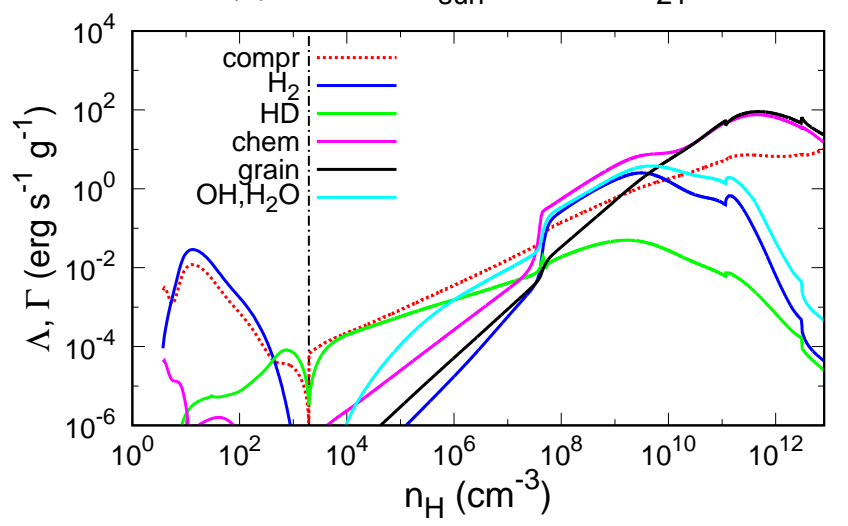

(d) $\mathrm{SN}, 10^{-2} \mathrm{Z}_{\text {sun }}, \mathrm{w} /$ dust, $\mathrm{J}_{21}=0$

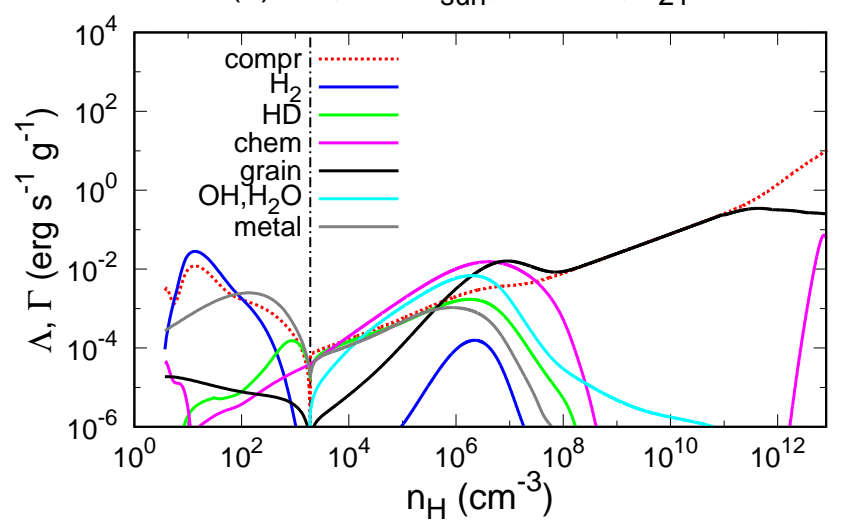

Figure 4. Same as Fig. 2, but for a cloud compressed by a SN shock, which is characterized by the initial density of $n_{\mathrm{H}, 1}=4 \mathrm{~cm}{ }^{-3}$.

inferred from the isobaric relation, $n_{\mathrm{H}, 1} T_{1} \simeq n_{\mathrm{frag}} T_{\mathrm{CMB}}$, as

$$
\begin{aligned}
M_{\mathrm{J}}\left(n_{\mathrm{frag}}, T_{\mathrm{CMB}}\right) \sim & 0.3 \mathrm{M}_{\odot}\left(\frac{T_{\mathrm{CMB}}}{10 \mathrm{~K}}\right)^{2}\left(\frac{\mu}{2}\right)^{-2} \\
& \times\left(\frac{n_{\mathrm{H}, 1}}{4000 \mathrm{~cm}^{-3}} \frac{T_{1}}{12000 \mathrm{~K}}\right)^{-1 / 2}
\end{aligned}
$$

and it becomes $\lesssim 3 \mathrm{M}_{\odot}$ if the initial density is $n_{\mathrm{H}, 1} \gtrsim$ $40 \mathrm{~cm}^{-3}\left(T_{\mathrm{CMB}} / \tilde{\sim} \mathrm{K}\right)^{4}$.

In the collapse phase of a clump, CMB effect appears explicitly in the models with dust and with $Z \gtrsim 10^{-3} \mathrm{Z}_{\odot}$ ( e.g., blue lines in Fig. 6c, d). In fact, the temperature remains below $30 \mathrm{~K}$ until the clump becomes optically thick to dust emission at $n_{\mathrm{H}} \sim 10^{12} \mathrm{~cm}^{-3}$. In particular, in the SN shock case, dust cooling triggers the re-fragmentation of a clump into $\sim 0.1 \mathrm{M}_{\odot}$ fragments at $n_{\mathrm{H}} \sim 10^{8} \mathrm{~cm}^{-3}$ (open triangle in Fig. 6d). This mass scale decreases as the CMB temperature decreases. In the other models, however, the temperature increases above $30 \mathrm{~K}$ and the thermal tracks soon converge with those of $T_{\mathrm{CMB}}=30 \mathrm{~K}$ models (cf. red and blue lines in Fig. 6). CMB effect is hardly observed in these cases. Note that in the CA model without dust and with $Z=10^{-2} \mathrm{Z}_{\odot}$ (the red line in Fig. 6c), owing to the rapid temperature increase at $n_{\mathrm{H}} \sim 10^{7}-10^{10} \mathrm{~cm}^{-3}$, the clump can collapse by accreting materials and elevating its mass from $\sim 0.3 \mathrm{M}_{\odot}$ to $\sim 10 \mathrm{M}_{\odot}$, i.e., the Jeans mass at
$\left(n_{\mathrm{H}}, T\right) \sim\left(10^{10} \mathrm{~cm}^{-3}, 10^{3} \mathrm{~K}\right)$. Therefore, the final clump mass is $M_{\text {clump }} \sim 10 \mathrm{M}_{\odot}$.

\section{FRAGMENT MASS}

Here we summarize the fragment mass scales in shockcompressed clouds, and discuss their dependence on the environmental conditions, i.e., initial metallicity, UV strength, and the presence of dust for both the CA and SN shocks. The condition for low-mass star formation is summarized in Section 4.2 for a wider range of initial densities.

\subsection{Dependence of fragment mass on metallicity, UV strength, and presence of dust}

In Fig. 7, the fragment mass scales are shown as a function of the initial metallicity for CA shock models with $J_{21}=0$ and $10^{4}$ (panels a and c), and SN shock models with $J_{21}=0$ and $10^{4}$ (panels b and $\mathrm{d}$ ). In each panel, blue and red colors indicate the clouds with and without dust. Open circles connected by solid lines indicate the clump mass $M_{\text {clump }}$. Asterisks and open triangles connected by dashed lines show the re-fragment mass scales $M_{\mathrm{re}-\text { frag }}$ determined by metal-line cooling and dust cooling, respectively. The grayshaded region in each panel marks the range of values where 
(a) $\mathrm{CA}, 10^{-4} \mathrm{Z}_{\text {sun }}$

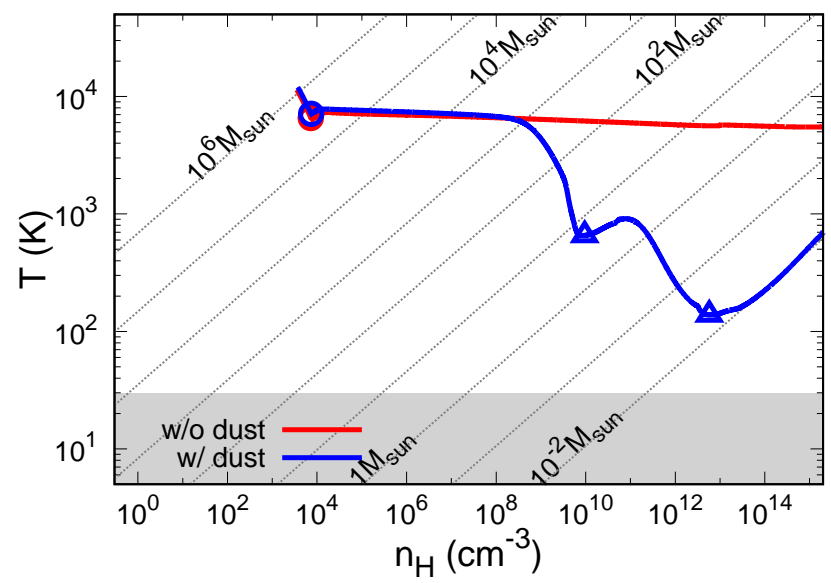

(c) $C A, 10^{-2} Z_{\text {sun }}$

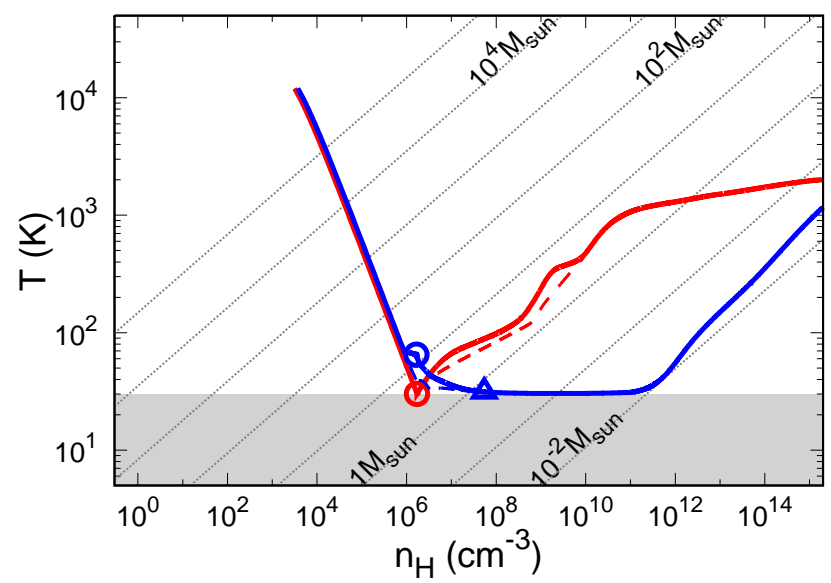

(b) $\mathrm{SN}, 10^{-4} \mathrm{Z}_{\text {sun }}$

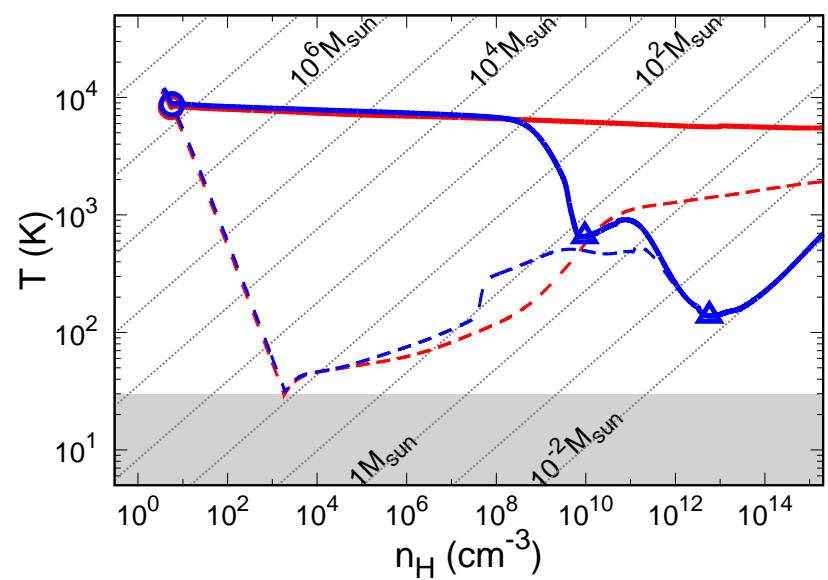

(d) $\mathrm{SN}, 10^{-2} \mathrm{Z}_{\text {sun }}$

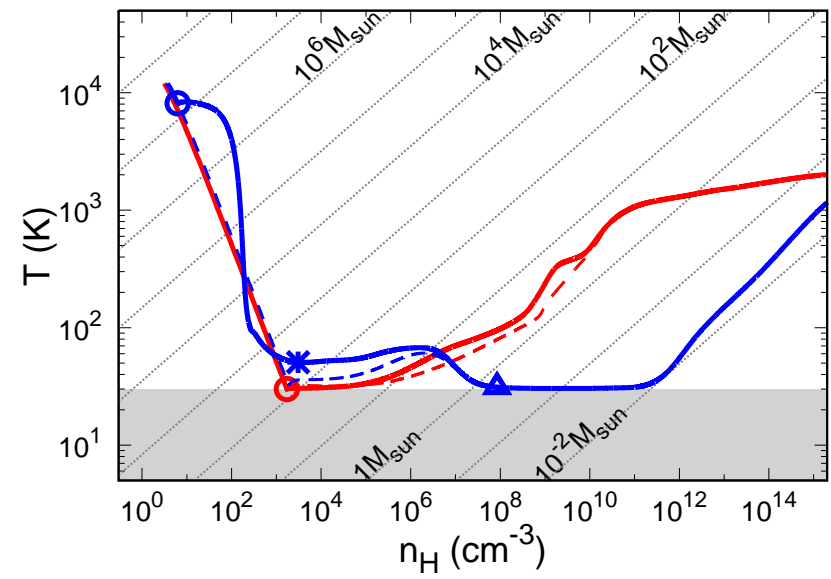

Figure 5. Same as Figs. 1 and 3, but showing the effects of UV irradiation on thermal evolution. In each panel, solid and dashed lines show the results with $J_{21}=10^{4}$ and $J_{21}=0$, respectively. Panels (a), (b), (c), and (d) refer to the models of $\left(\mathrm{CA}\right.$, $\left.10^{-4} \mathrm{Z}_{\odot}\right)$, (SN, $\left.10^{-4} \mathrm{Z}_{\odot}\right),\left(\mathrm{CA}, 10^{-2} \mathrm{Z}_{\odot}\right)$, and (SN, $\left.10^{-2} \mathrm{Z}_{\odot}\right)$, respectively. On thermal tracks, open circles indicate the epochs of clump formation and asterisks and open triangles indicate those of re-fragmentation.

$M_{\text {frag }} \leq 3 \mathrm{M}_{\odot}$ and sub-solar mass stars form when the star formation efficiency is $\simeq 30 \%$. The star formation efficiency is observationally estimated to be in the range of $\simeq 20-40 \%$, by comparing the shape and amplitude of the prestellar core mass function with those of the stellar initial mass function (e.g., Alves et al. 2007; André et al. 2010). Using threedimensional resistive magneto-hydrodynamical (MHD) simulations, Machida \& Matsumoto (2012) suggested that less than half of the mass of a prestellar cloud becomes a star and the rest is released into the interstellar space by a protostellar outflow.

We first discuss the results for CA shocks (Fig. 7a, c). Owing to the high initial density, the thermal evolution and thus the fragment mass scales (both $M_{\text {clump }}$ and $M_{\text {re-frag }}$ ) are hardly affected by UV irradiation for most of the models (see also Section 3.3). The clump mass $M_{\text {clump }}$ (open circle) is almost entirely determined by the gas metallicity: it is $\sim 10^{6} \mathrm{M}_{\odot}$ for metallicities lower than $\sim 10^{-4} \mathrm{Z}_{\odot}$ and decreases abruptly to $\simeq 10 \mathrm{M}_{\odot}$ for $Z \gtrsim 10^{-3} \mathrm{Z}_{\odot}$. The pres- ence of dust increases (lowers) the clump mass $M_{\text {clump }}$ only slightly for $Z=10^{-3} \mathrm{Z}_{\odot}\left(10^{-2} \mathrm{Z}_{\odot}\right.$, respectively). In models with dust (open triangles), these clumps re-fragment into sub-solar mass pieces and low-mass star formation becomes possible when $10^{-5} \lesssim Z / \mathrm{Z}_{\odot} \lesssim 10^{-3}$ for the $J_{21}=0$ case and $Z / Z_{\odot} \gtrsim 10^{-5}$ for the $J_{21}=10^{4}$ case, respectively.

In $\mathrm{SN}$ shock models (Fig. 7b, d), due to the low initial density, the thermal evolution and fragment mass scale are greatly affected by UV irradiation. In the absence of UV irradiation (Fig. 7b, open circles), the clump mass is $M_{\text {clump }} \simeq 200 \mathrm{M}_{\odot}$ at all initial metallicities. Under the strong UV field of $J_{21}=10^{4}$ (Fig. $7 \mathrm{~d}$, open circles), the clump mass is boosted to $M_{\text {clump }} \sim 10^{7} \mathrm{M}_{\odot}$. The only exception is the model with $Z=10^{-2} \mathrm{Z}_{\odot}$ and no dust, where the gas can cool isobarically to $T_{\mathrm{CMB}}$ by metal-line cooling and no re-fragmentation is expected thereafter, so that the clump mass $M_{\text {clump }}$ is the same as that in the $J_{21}=0$ case. In SN shock models with $Z \gtrsim 10^{-3} \mathrm{Z}_{\odot}$, although metal-line cooling triggers re-fragmentation of clumps, the fragment mass 
(a) $\mathrm{CA}, 10^{-4} \mathrm{Z}_{\text {sun }}, \mathrm{J}_{21}=0$

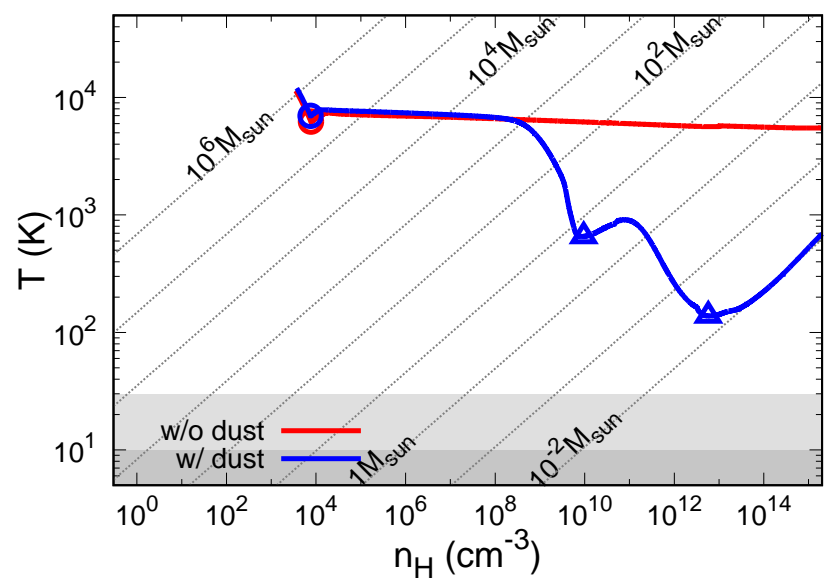

(c) $C A, 10^{-2} Z_{\text {sun }}, J_{21}=0$

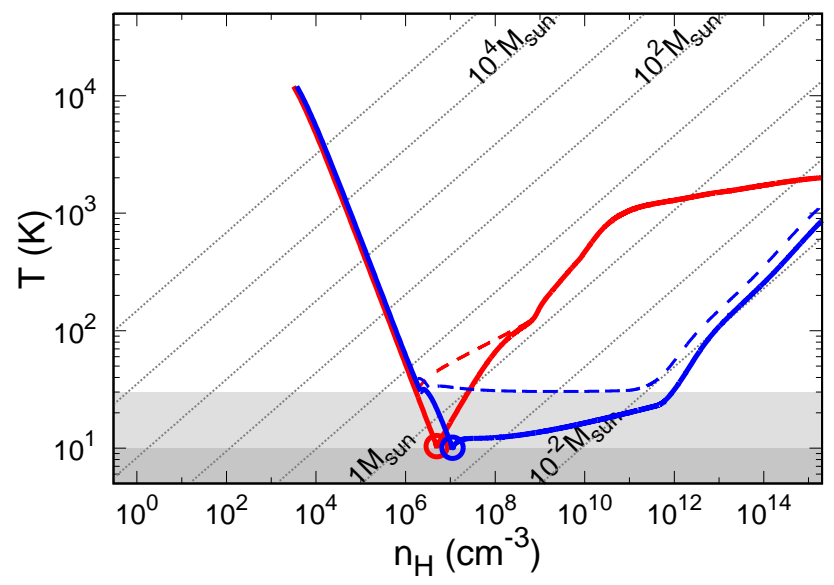

(b) $\mathrm{SN}, 10^{-4} \mathrm{Z}_{\text {sun }}, \mathrm{J}_{21}=0$

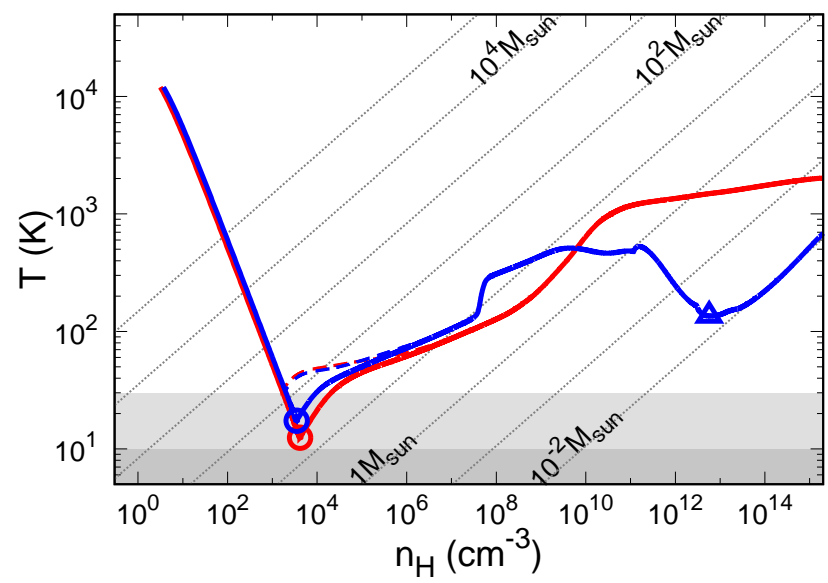

(d) $\mathrm{SN}, 10^{-2} \mathrm{Z}_{\text {sun }}, \mathrm{J}_{21}=0$

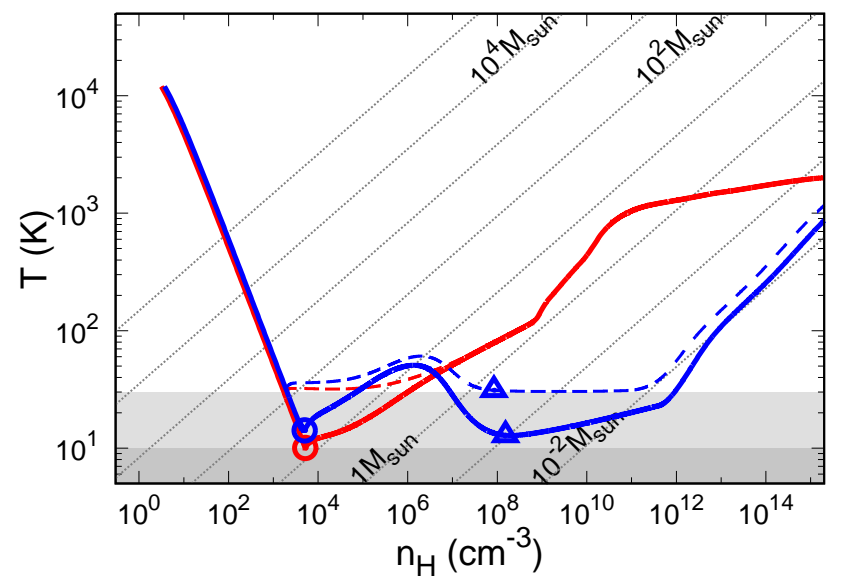

Figure 6. Same as Fig. 5, but showing the CMB effects. Solid and dashed lines show the results with $T_{\mathrm{CMB}}=10 \mathrm{~K}$ and $T_{\mathrm{CMB}}=30 \mathrm{~K}$, respectively.

$M_{\text {re-frag }}$ is higher than $\sim 30 \mathrm{M}_{\odot}$ (asterisks in Fig. $7 \mathrm{~d}$ ). In the models with dust, these clumps fragment again at high enough densities $\left(\gtrsim 10^{8} \mathrm{~cm}^{-3}\right)$ thanks to dust cooling and so the fragment mass $M_{\mathrm{re}-\text { frag }}$ can be very small $\left(\simeq 0.01-1 \mathrm{M}_{\odot}\right)$ when $Z \gtrsim 10^{-5} \mathrm{Z}_{\odot}$ (open triangles), comparable to $\mathrm{CA}$ shock cases.

\subsection{Condition for low-mass star formation}

Here we discuss the final fragment mass in the collapse phase, which is given by $M_{\text {frag }}=\min \left(M_{\text {clump }}, M_{\text {re-frag }}\right)$, for a wider range of initial densities $n_{\mathrm{H}, 1}=1-10^{5} \mathrm{~cm}^{-3}$ and metallicities $Z=0-10^{-2} \mathrm{Z}_{\odot}$. Fig. 8 shows models of $J_{21}=0$ without and with dust (panels a and b), and $J_{21}=$ $10^{4}$ without and with dust (panels $\mathrm{c}$ and $\mathrm{d}$ ). Each panel is divided into five domains of $\left(Z, n_{\mathrm{H}, 1}\right)$, depending on the value of $M_{\text {frag }}$ : (i) very low-mass $\left(M_{\text {frag }}<0.3 \mathrm{M}_{\odot}\right.$; grey shaded, open circles), (ii) low-mass $\left(\sim 0.3-3 \mathrm{M}_{\odot}\right.$; cyan shaded, filled circles), (iii) intermediate mass $\left(\sim 3-30 \mathrm{M}_{\odot}\right.$; green shaded, triangles), (iv) massive $\left(\sim 30-3000 \mathrm{M}_{\odot}\right.$; yellow shaded, squares), and (v) very massive $\left(>10^{5} \mathrm{M}_{\odot}\right.$; red shaded, crosses) fragments. The CMB temperature is set at $T_{\mathrm{CMB}}=30 \mathrm{~K}$.

In all the models without dust (Fig. 8a, c), the fragment mass is higher than $\sim 3 \mathrm{M}_{\odot}$ and there is no parameter space forming low-mass fragments. When $J_{21}=0$ (Fig. 8a) and $n_{\mathrm{H}, 1} \lesssim 100 \mathrm{~cm}^{-3}$, massive fragments (in the $30-3000 \mathrm{M}_{\odot}$ range) form, and this value depends on the initial metallicity only weakly. In models with $n_{\mathrm{H}, 1} \gtrsim 10^{3} \mathrm{~cm}^{-3}$, the fragment mass scale bifurcates into the intermediate scale range $\left(3-30 \mathrm{M}_{\odot}\right)$ for $Z \gtrsim 10^{-4} \mathrm{Z}_{\odot}$ and the very massive scale range $\left(\gtrsim 10^{5} \mathrm{M}_{\odot}\right)$ for $Z \lesssim 10^{-4} \mathrm{Z}_{\odot}$. The UV irradiation greatly elevates the fragment mass scale from the massive to the very massive scale in models with $Z \lesssim 10^{-4} \mathrm{Z}_{\odot}$ and $n_{\mathrm{H}, 1} \lesssim 10^{3} \mathrm{~cm}^{-3}$, but its effect is not observed in other models (Fig. 8c). In the models with dust (Fig. 8b, d), as a result of clump re-fragmentation, very low-mass $\left(<0.3 \mathrm{M}_{\odot}\right)$ and low-mass $\left(0.3-3 \mathrm{M}_{\odot}\right)$ fragments are formed for $10^{-5} \lesssim$ $Z / \mathrm{Z}_{\odot} \lesssim 10^{-3}$ and $Z / \mathrm{Z}_{\odot}=10^{-2}$, respectively. This trend is observed in almost all the models with dust. In conclusion, 
(a) $\mathrm{CA}, \mathrm{J}_{21}=0$

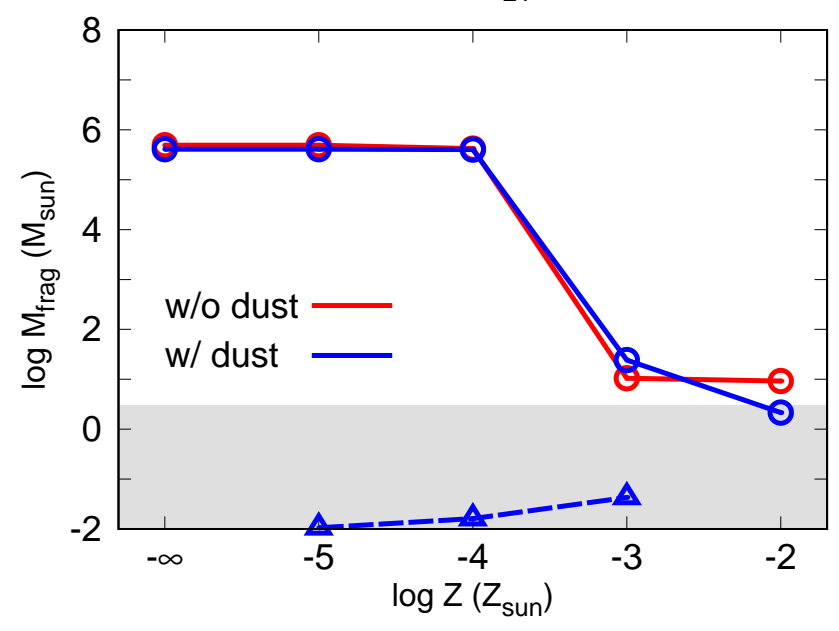

(c) $\mathrm{CA}, \mathrm{J}_{21}=10^{4}$

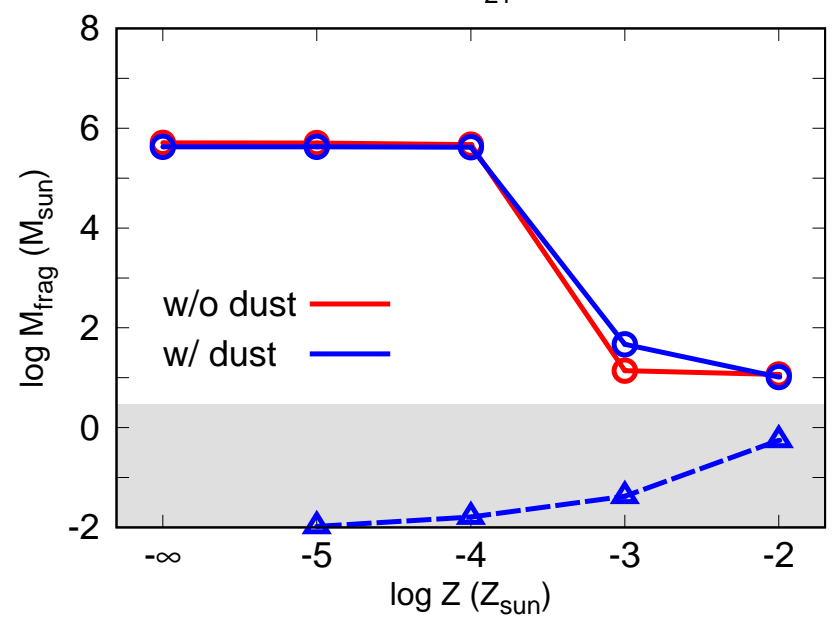

(b) $\mathrm{SN}, \mathrm{J}_{21}=0$

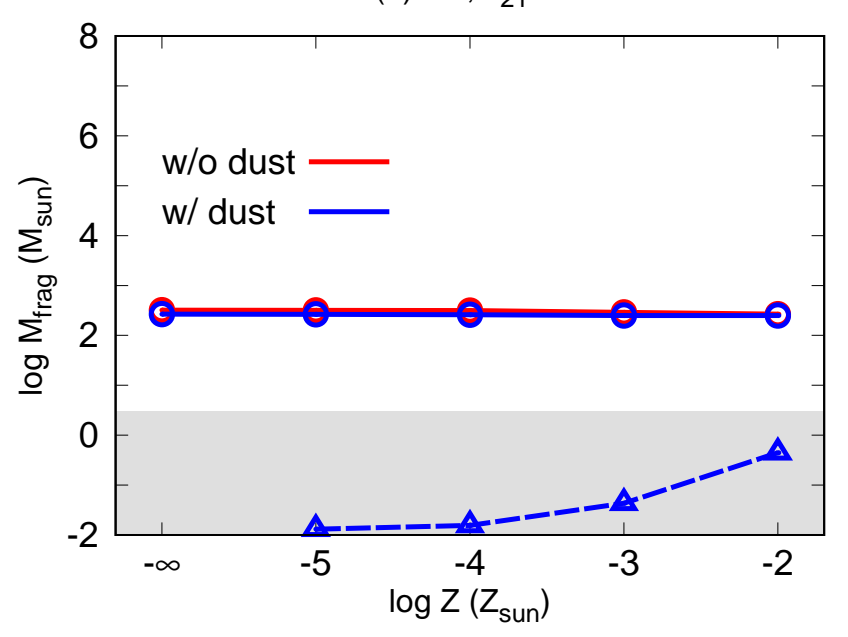

(d) $\mathrm{SN}, \mathrm{J}_{21}=10^{4}$

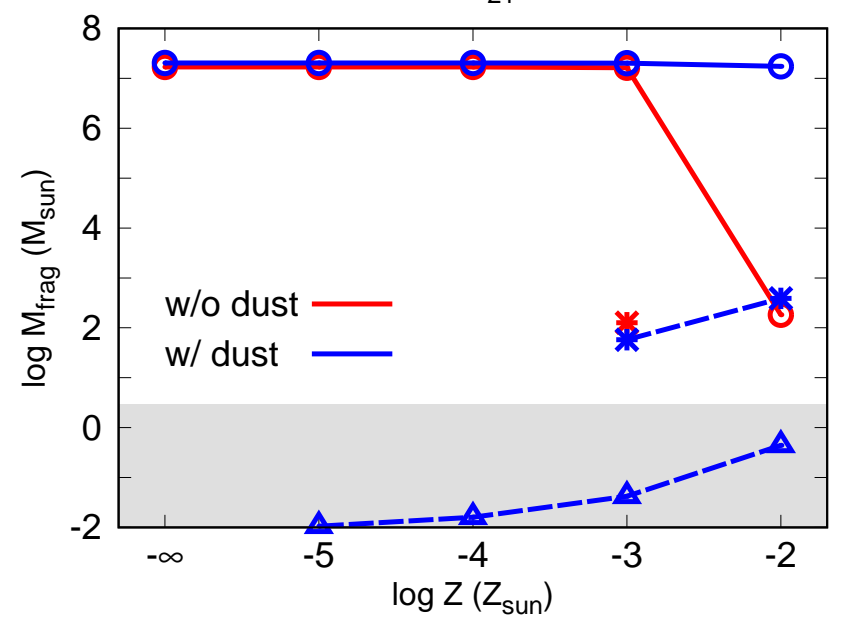

Figure 7. Fragment mass as a function of metallicity, and its dependence on the initial density, UV strength, and the presence of dust. Panels (a), (b), (c), and (d) refer to the models of $\left(\mathrm{CA}, J_{21}=0\right),\left(\mathrm{SN}, J_{21}=0\right),\left(\mathrm{CA}, J_{21}=10^{4}\right)$, and $\left(\mathrm{SN}, J_{21}=10^{4}\right)$, respectively. In each panel, blue and red colors indicate the clouds with and without dust. Open circles connected by solid lines, and asterisks and open triangles connected by dashed lines are used to identify the fragment mass scales of $M_{\text {clump }}$, and $M_{\mathrm{re}-\text { frag, }}$, respectively. Sub-solar mass stars are expected to form in the gray-shaded regions (see text).

the presence of dust is indispensable for the formation of sub-solar mass stars also in shock-compressed clouds.

\section{SUMMARY AND DISCUSSION}

We have studied the thermal evolution of shock-compressed clouds starting from a temperature of $\sim 10^{4} \mathrm{~K}$, for a large range of metallicities $Z=0-10^{-2} \mathrm{Z}_{\odot}$, post-shock densities $n_{\mathrm{H}, 1}=1-10^{5} \mathrm{~cm}^{-3}$, and $\mathrm{CMB}$ temperatures $T_{\mathrm{CMB}}=10$ and $30 \mathrm{~K}$, with and without dust or external UV fields. The calculation is based on a one-zone model equipped with detailed thermal and chemical processes. In particular, two kinds of shocks have been examined in some detail: (i) coldaccretion shock $\left(n_{\mathrm{H}, 1}=4 \times 10^{3} \mathrm{~cm}^{-3}\right)$ and (ii) supernova shock $\left(n_{\mathrm{H}, 1}=4 \mathrm{~cm}^{-3}\right)$. Based on the obtained temperature evolution, we have estimated the fragmentation mass scales and discussed the condition for subsolar-mass star formation. Our major findings can be summarized as follows:

- Shock-compressed clouds contract isobarically via atomic or molecular line cooling, until self-gravitating clumps are produced by fragmentation. In the subsequent collapse phase, the temperature of a clump is increased due to compressional heating as well as $\mathrm{H}_{2}$ formation heating, while the density increases by a few orders of magnitude.

- In models without dust, no further fragmentation of a clump is expected during the collapse phase. The clump mass is higher than $3 \mathrm{M}_{\odot}$ in all cases (Fig. 8a, c).

- The presence of dust hardly changes the thermal evolution and the clump mass at fragmentation in the isobaric phase. In the collapse phase, dust cooling enables these clumps to fragment again into sub-solar mass dense cores, 
(a) w/o dust, $\mathrm{J}_{21}=0, \mathrm{~T}_{\mathrm{CMB}}=30 \mathrm{~K}$

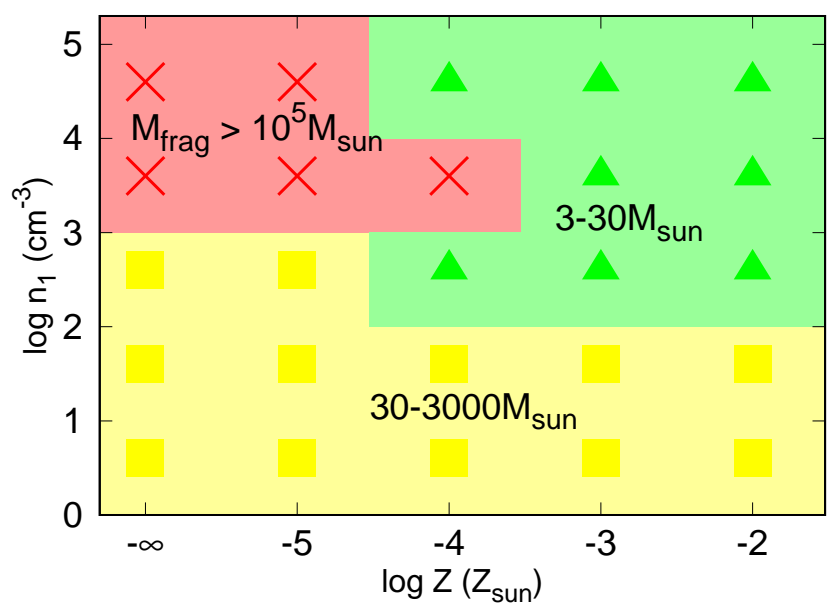

(c) w/o dust, $\mathrm{J}_{21}=10^{4}, \mathrm{~T}_{\mathrm{CMB}}=30 \mathrm{~K}$

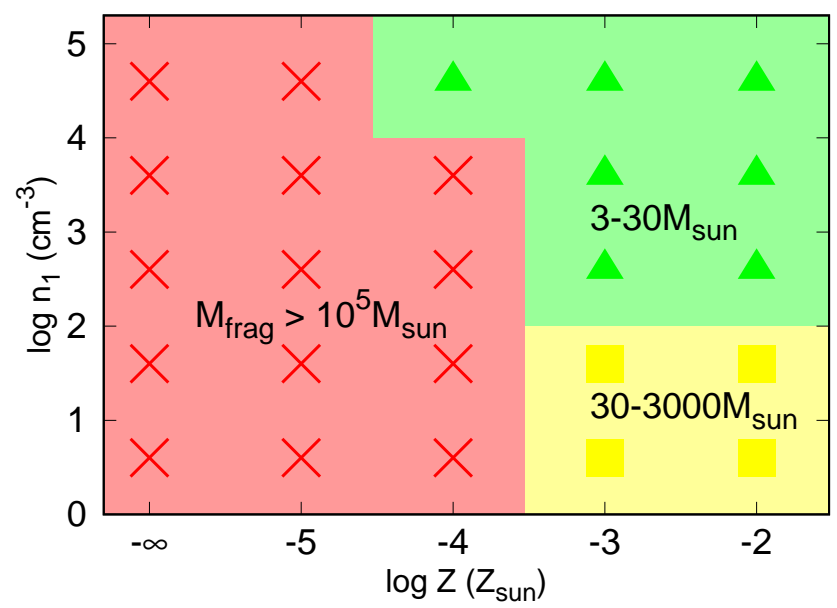

(b) w/ dust, $\mathrm{J}_{21}=0, \mathrm{~T}_{\mathrm{CMB}}=30 \mathrm{~K}$

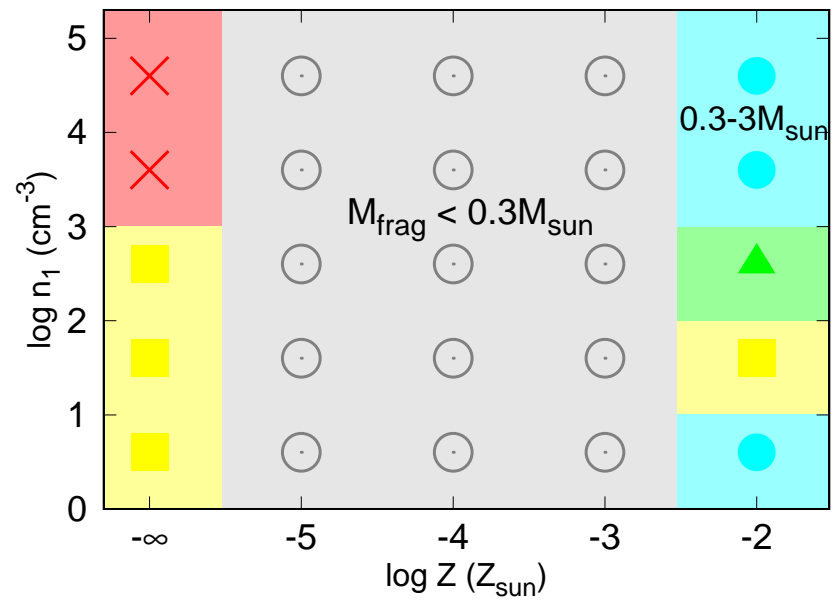

(d) $\mathrm{w} /$ dust, $\mathrm{J}_{21}=10^{4}, \mathrm{~T}_{\mathrm{CMB}}=30 \mathrm{~K}$

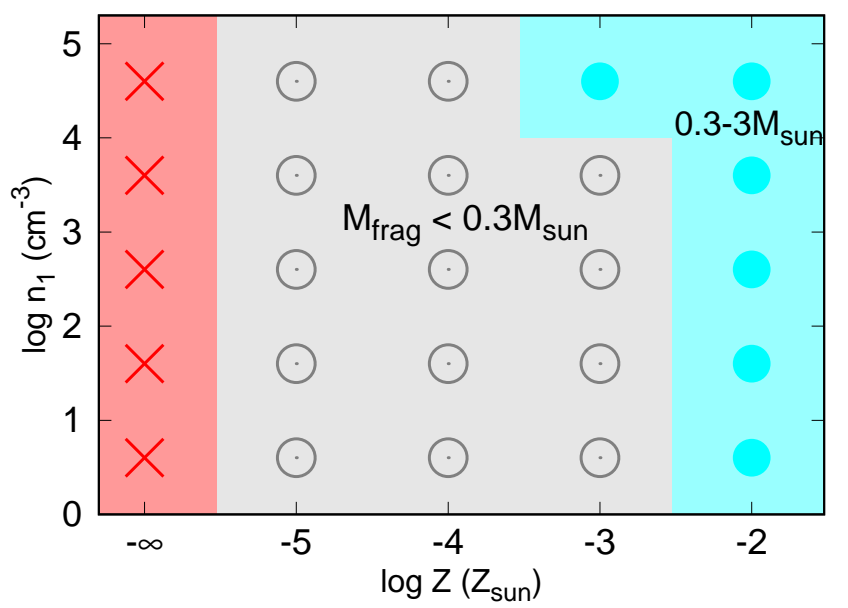

Figure 8. Conditions for the formation of very low mass $\left(M_{\text {frag }}<0.3 \mathrm{M}_{\odot}\right.$; grey shaded, open circles), low mass $\left(M_{\text {frag }}=0.3-3 \mathrm{M}_{\odot}\right.$; cyan shaded, filled circles $)$, intermediate mass $\left(M_{\text {frag }}=3-30 \mathrm{M}_{\odot}\right.$; green shaded, triangles $)$, massive $\left(M_{\text {frag }}=30-3000\right.$ M $\odot ;$ yellow shaded, squares), and very massive $\left(M_{\text {frag }}>10^{5} \mathrm{M}_{\odot}\right.$; red shaded, crosses $)$ fragments. In this plot, $M_{\text {frag }}$ means the minimum fragment mass during the evolution, i.e., $M_{\text {frag }}=\min \left[M_{\text {clump }}, M_{\text {re-frag }}\right]$. Panels (a) and (b) refer to models of $J_{21}=0$ without and with dust, and panels (c) and (d) refer to models of $J_{21}=10^{4}$ without and with dust, respectively.

as long as the metallicity is higher than $\sim 10^{-5} \mathrm{Z}_{\odot}$ (Fig. 8b, d).

- UV irradiation affects the thermal evolution and clump mass considerably for the models with a low initial density, as in SN shocks. In some models with enough metals $\left(Z \gtrsim 10^{-3} Z_{\odot}\right)$, the clump experiences another episode of fragmentation by metal-line cooling during the collapse phase. This, however, only produces fragments as massive as $30-10^{3} \mathrm{M}_{\odot}$ (asterisks in Fig. $7 \mathrm{~d}$ ).

- Even if a cloud starts contraction with different initial densities or UV strengths, thermal tracks converge into a common one for each metallicity when the density is high enough for dust cooling to become effective, inducing refragmentation of a clump into sub-solar mass dense cores. Therefore, the mass scale of a dense core is controlled by the amount of dust.
The evolution and fragmentation of shock-compressed clouds have also been studied by Safranek-Shrader et al. (2010), for the case of a CA flow without considering dust grains, using a semi-analytical model similar to ours. They find that when the metallicity becomes higher than $\simeq 10^{-4}-10^{-3} \mathrm{Z}_{\odot}$, the fragment mass shows a sharp drop from $\simeq 10^{5} \mathrm{M}_{\odot}$ to $<100 \mathrm{M}_{\odot}$, which agrees with our results (the red lines in Fig. 7a, c). They also find that $\lesssim 3 \mathrm{M}_{\odot}$ fragments form when $Z \geq 10^{-2.5} \mathrm{Z}_{\odot}$, while we have shown that the fragment mass never becomes lower than $\simeq 10 \mathrm{M}_{\odot}$ in the absence of dust. This difference comes from the fact that they do not follow the evolution after fragmentation. In the absence of dust, the fragment contracts while raising its temperature so rapidly that the instantaneous Jeans mass exceeds the fragment mass. In this case, the fragment contracts in a quasi-static manner by accreting surrounding materials and keeping its mass equal to the instantaneous Jeans 
mass. This boosts the fragment mass to the maximum value of the instantaneous Jeans mass during evolution, which is $\simeq 10 \mathrm{M}_{\odot}$ when $Z \geq 10^{-2.5} \mathrm{Z}_{\odot}$.

Our results are based on a simple one-zone model, and any multi-dimensional effect like rotation and turbulence may modify our conclusions. Inoue \& Omukai (2015) studied the thermal evolution of shock-compressed metal-poor clouds using three-dimensional (3D) hydrodynamical simulations. They find that the shock-compressed layer becomes highly turbulent and inhomogeneous both in density and temperature, owing to the growth of thermal instabilities. Their study was, however, unable to follow the evolution up to the point where gravitationally unstable fragments form. 3D hydrodynamical calculations including self-gravity are needed to study the multi-dimensional effects on the fragment mass scales.

Magnetic fields may have played an important role in star formation, also in early galaxies. Inoue \& Inutsuka $(2008,2009,2012)$ studied, in the context of present-day star-formation, the effect of magnetic fields on the evolution of a shock-compressed layer, both by 2D and 3D magnetohydrodynamical (MHD) simulations. The result depends on the angle between the magnetic field and the flow direction in the following way. If the field line is perpendicular to the flow direction, the magnetic pressure balances with the ram pressure after weak compression. The shocked gas is then hardly compressed and cools isochorically instead, lowering its pressure. The fragment mass, calculated by the effective Jeans mass, is boosted by lower fragmentation densities as well as by the contribution of magnetic and turbulent pressures. On the other hand, if the magnetic field and the flow direction are parallel to each other, the magnetic pressure does not work against compression, so that it will have little effect on the thermal evolution and fragmentation. In order to see if such magnetic effects are operating in lowmetallicity clouds, similar multi-dimensional MHD calculations are needed.

Extremely metal-poor (EMP) stars observed in the Galactic halo provide valuable information about the birthplace of first low-mass stars. Schneider et al. (2012b) analyzed the origin of an EMP star SDSS J1029+1729 with a total metallicity of $\sim 4.5 \times 10^{-5} \mathrm{Z}_{\odot}$, discovered by Caffau et al. (2011). They concluded that this star was formed in a cloud containing both metals and dust grains released by a Pop III SN of $20-40 \mathrm{M}_{\odot}$. Although they considered a parent cloud contracting without shock-compression, we find that this star can also be formed in a shock-compressed cloud. More recently, Aguado et al. (2018) reported the discovery of a mega metal-poor star SDSS J0023+0303 with the iron abundance of $[\mathrm{Fe} / \mathrm{H}]<-6.6$. Only the upper limit is obtained for the carbon abundance $([\mathrm{C} / \mathrm{H}]<-2.1)$. If there is indeed no carbon enhancement, the total metallicity is extremely low $\left(<10^{-6.6} \mathrm{Z}_{\odot}\right)$ and dust-driven fragmentation considered here does not operate. In such a case, we have to invoke another pathway for low-mass star formation. Once a protostar is formed, it gains mass through the accretion from the circumstellar disc. A multiple protostar system might be formed by disc fragmentation and some of the satellite protostars would be ejected from the system through multibody gravitational interaction. If the growth of a protostar is stunted before its mass exceeds $\sim \mathrm{M}_{\odot}$, a low-mass star can form even in the zero metal case (Clark et al. 2011;
Greif et al. 2012; Susa et al. 2014; Chiaki et al. 2016). This provides a possible pathway for the formation of the star SDSS J0023+0303.

\section{ACKNOWLEDGMENTS}

We thank Drs Hide Yajima, Kazu Sugimura, and Gen Chiaki for fruitful discussions. Numerical calculations are performed by the computer cluster, Draco, supported by the Frontier Research Institute for Interdisciplinary Sciences in Tohoku University. This work is supported in part by MEXT/JSPS KAKENHI grants (DN:16J02951, KO:25287040, 17H01102, 17H02869). The research leading to these results has received funding from the European Research Council under the European Union's Seventh Framework Programme (FP/2007-2013)/ERC Grant Agreement n. 306476.

\section{REFERENCES}

Abel T., Bryan G. L., Norman M. L., 2002, Science, 295, 93 Abrahamsson E., Krems R. V., Dalgarno A., 2007, ApJ, 654, 1171

Aguado D. S., Allende Prieto C., González Hernández J. I., Rebolo R., 2018, ApJL, 854, L34

Alves J., Lombardi M., Lada C. J., 2007, A\&A, 462, L17

Anders E., Grevesse N., 1989, GeCoA, 53, 197

André P., et al., 2010, A\&A, 518, L102

Bastian N., Covey K. R., Meyer M. R., 2010, ARA\&A, 48, 339

Beers T. C., Christlieb N., 2005, ARA\&A, 43, 531

Bovino S., Grassi T., Schleicher D. R. G., Banerjee R., 2016, ApJ, 832,154

Bromm V., Loeb A., 2003, Nature, 425, 812

Bromm V., Ferrara A., Coppi P. S., Larson R. B., 2001, MNRAS, 328, 969

Bromm V., Coppi P. S., Larson R. B., 2002, ApJ, 564, 23

Caffau E., et al., 2011, Nature, 477, 67

Chiaki G., Yoshida N., Kitayama T., 2013, ApJ, 762, 50

Chiaki G., Yoshida N., Hirano S., 2016, MNRAS, 463, 2781

Clark P. C., Glover S. C. O., Klessen R. S., 2008, ApJ, 672, 757

Clark P. C., Glover S. C. O., Smith R. J., Greif T. H., Klessen R. S., Bromm V., 2011, Science, 331, 1040

Dopcke G., Glover S. C. O., Clark P. C., Klessen R. S., 2013, ApJ, 766, 103

Elmegreen B. G., Elmegreen D. M., 1978, ApJ, 220, 1051

Frebel A., Norris J. E., 2015, ARA\&A, 53, 631

Galli D., Palla F., 2013, ARA\&A, 51, 163

Ginolfi M., Graziani L., Schneider R., Marassi S., Valiante R., Dell'Agli F., Ventura P., Hunt L. K., 2018, MNRAS, 473, 4538

Glover S. C. O., Clark P. C., 2014, MNRAS, 437, 9

Greif T. H., Johnson J. L., Klessen R. S., Bromm V., 2008, MNRAS, 387, 1021

Greif T. H., Bromm V., Clark P. C., Glover S. C. O., Smith R. J., Klessen R. S., Yoshida N., Springel V., 2012, MNRAS, 424,399

Habing H. J., 1968, BAN, 19, 421

Hirano S., Hosokawa T., Yoshida N., Umeda H., Omukai K., Chiaki G., Yorke H. W., 2014, ApJ, 781, 60

Hosokawa T., Yoshida N., Omukai K., Yorke H. W., 2012, ApJL, 760, L37

Inayoshi K., Omukai K., 2012, MNRAS, 422, 2539

Inayoshi K., Omukai K., Tasker E., 2014, MNRAS, 445, L109

Inoue T., Inutsuka S.-i., 2008, ApJ, 687, 303

Inoue T., Inutsuka S.-i., 2009, ApJ, 704, 161

Inoue T., Inutsuka S.-i., 2012, ApJ, 759, 35 
Inoue T., Omukai K., 2015, ApJ, 805, 73

Jappsen A.-K., Glover S. C. O., Klessen R. S., Mac Low M.-M., 2007, ApJ, 660, 1332

Kitayama T., Yoshida N., 2005, ApJ, 630, 675

Kroupa P., 2002, Science, 295, 82

Larson R. B., 1969, MNRAS, 145, 271

Machida M. N., Matsumoto T., 2012, MNRAS, 421, 588

Marassi S., Schneider R., Limongi M., Chieffi A., Bocchio M., Bianchi S., 2015, MNRAS, 454, 4250

Mathis J. S., Mezger P. G., Panagia N., 1983, A\&A, 128, 212

Nagakura T., Hosokawa T., Omukai K., 2009, MNRAS, 399, 2183

Nakauchi D., Inayoshi K., Omukai K., 2014, MNRAS, 442, 2667

Nakauchi D., Hosokawa T., Omukai K., Saio H., Nomoto K., 2017, MNRAS, 465, 5016

Omukai K., 2000, ApJ, 534, 809

Omukai K., 2012, PASJ, 64, 114

Omukai K., Tsuribe T., Schneider R., Ferrara A., 2005, ApJ, 626,627

Omukai K., Schneider R., Haiman Z., 2008, ApJ, 686, 801

Omukai K., Hosokawa T., Yoshida N., 2010, ApJ, 722, 1793

Penston M. V., 1969, MNRAS, 144, 425

Pollack J. B., Hollenbach D., Beckwith S., Simonelli D. P., Roush T., Fong W., 1994, ApJ, 421, 615

Safranek-Shrader C., Bromm V., Milosavljević M., 2010, ApJ, 723,1568

Safranek-Shrader C., Milosavljević M., Bromm V., 2014, MNRAS, 438, 1669

Safranek-Shrader C., Montgomery M. H., Milosavljević M., Bromm V., 2016, MNRAS, 455, 3288

Santoro F., Shull J. M., 2006, ApJ, 643, 26

Schneider R., Omukai K., 2010, MNRAS, 402, 429

Schneider R., Ferrara A., Natarajan P., Omukai K., 2002, ApJ, 571,30

Schneider R., Ferrara A., Salvaterra R., Omukai K., Bromm V., 2003, Nature, 422, 869

Schneider R., Omukai K., Inoue A. K., Ferrara A., 2006, MNRAS, 369, 1437

Schneider R., Omukai K., Bianchi S., Valiante R., 2012a, MNRAS, 419, 1566

Schneider R., Omukai K., Limongi M., Ferrara A., Salvaterra R., Chieffi A., Bianchi S., 2012b, MNRAS, 423, L60

Shapiro P. R., Kang H., 1987, ApJ, 318, 32

Smith B. D., Turk M. J., Sigurdsson S., O'Shea B. W., Norman M. L., 2009, doi:10.1088/0004-637X/691/1/441, 691, 441

Sugimura K., Mizuno Y., Matsumoto T., Omukai K., 2017, MNRAS, 469, 4022

Susa H., Hasegawa K., Tominaga N., 2014, ApJ, 792, 32

Tsuribe T., Omukai K., 2006, ApJ, 642, L61

Tsuribe T., Omukai K., 2008, ApJ, 676, L45

Wise J. H., Abel T., 2007, ApJ, 665, 899

Wise J. H., Turk M. J., Abel T., 2008, ApJ, 682, 745

Yamada M., Nishi R., 1998, ApJ, 505, 148

Yoshida N., Omukai K., Hernquist L., 2008, Science, 321, 669

de Bennassuti M., Schneider R., Valiante R., Salvadori S., 2014, MNRAS, 445, 3039

de Bennassuti M., Salvadori S., Schneider R., Valiante R., Omukai K., 2017, MNRAS, 465, 926

This paper has been typeset from a $\mathrm{T}_{\mathrm{E}} \mathrm{X} / \mathrm{LAT}_{\mathrm{E}} \mathrm{X}$ file prepared by the author. 